\title{
Probabilistic Control of Nonlinear Uncertain Systems
}

\author{
Qian Wang $^{1}$ and Robert F. Stengel ${ }^{2}$ \\ 1 Mechanical Engineering, Penn State University, University Park, PA 16802, \\ quw6@psu.edu \\ 2 Mechanical and Aerospace Engineering, Princeton University, Princeton, NJ \\ 08544, stengel@princeton.edu
}

Summary. Robust controllers for nonlinear systems with uncertain parameters can be reliably designed using probabilistic methods. In this chapter, a design approach based on the combination of stochastic robustness and dynamic inversion is presented for general systems that have a feedback-linearizable nominal system. The efficacy of this control approach is illustrated through the design of flight control systems for a hypersonic aircraft and a highly nonlinear, complex aircraft model. The proposed stochastic robust nonlinear control explores the direct design of nonlinear flight control logic; therefore the final design accounts for all significant nonlinearities in the aircraft's high-fidelity simulation model. Monte Carlo simulation is used to estimate the likelihood of closed-loop system instability and violation of performance requirements subject to variations of the probabilistic system parameters. The stochastic robustness cost function is defined in terms of the probabilities that design criteria will not be satisfied. We use randomized algorithms, in particular genetic algorithms, to search the design parameters of the parameterized controller with feedback linearization structure. The design approach is an extension of earlier methods for probabilistic robust control of linear systems. Prior results are reviewed, and the nonlinear approach is presented.

\section{Introduction}

Control systems should be designed to run satisfactorily not only with assumed plant parameters but with possible variations in operating conditions. Perfect models of systems to be controlled are rarely available when controllers are being designed, parameters of similar plants are likely to vary from one example to the next, and dynamic characteristics may change as parts wear or operating points shift. Control system designs must be tolerant of these differences for practical control to take place, that is, they must be robust. For parametric uncertainty, guaranteed stability-bound estimates often are unduly conservative, and the resulting controller usually needs very high control effort. With respect to computational complexity, many worst-case 
deterministic robust control problems are proved to be NP hard. If instead of worst-case guaranteed conclusions, probabilistic robustness is acceptable, computational complexity can be reduced significantly. In probabilistic robust control design, randomized algorithms with polynomial complexity are used to characterize system robustness and to identify satisfactory controllers.

Many problems in system synthesis can be formulated as the minimization of an objective function with respect to the parameters of a parameterized controller. The probabilistic robust control problem is transformed to a stochastic optimization problem. Combinations of a variety of pre-existing control methodologies and the probabilistic approach to robustness have been applied to control designs such as Linear-Quadratic-Gaussian regulators [31, 41], transfer function sweep designs [57], quadratic stabilization for linear systems [7], robust Linear Matrix Inequality (LMI) or Quadratic Matrix Inequality (QMI) [9], Linear-Parameter-Varying control [15], robust $H_{2}$ control [26] and Model Predictive control [21].

The probabilistic approach is readily applied to nonlinear designs as well as to linear designs. We present a framework for nonlinear robust control that merges the stochastic approach with feedback linearization. There has been intensive research in deterministic nonlinear robust control using, for example, Lyapunov redesign, backstepping, sliding-mode control, and neural network based adaptive robust control [18]. The probabilistic approach to control design could reduce design conservativeness significantly, and it provides a viable treatment for system robustness with respect to uncertain parameters that may enter the system in an arbitrary way. In this chapter, the proposed stochastic feedback linearization approach is illustrated through two flight control applications. The first application is to the control of the longitudinal motion of a NASA Langley hypersonic aircraft [49] cruising at a Mach number of 15 and at an altitude of 110,000ft. There are twenty-eight uncertain parameters in characterizing the aircraft's inertial and aerodynamic model. Robustness metrics include system stability and thirty-eight performance specifications for velocity and altitude command responses in the presence of uncertain parameter variations. The probabilistic robust control design is formulated as a stochastic optimization of a cost function that is a weighted quadratic sum of these probabilities of violation of design specifications. Due to the non-convex and non-deterministic nature of this stochastic optimization problem, genetic algorithms are used here to search the controller parameters. We apply a similar approach to the probabilistic robust control of the six-degree-of-freedom motion of a High Incidence Research Model (HIRM) [28] whose highly nonlinear aerodynamic model is described by a combination of analytic equations and look-up tables. Due to the complexity of the system model, a two-timescale decomposition is used in the design of controller structure. The resulted nonlinear control design is evaluated and compared against existing designs with respect to handling qualities for a wide range of flight envelope and in the presence of system parametric uncertainties. 
This Chapter is organized as follows: Section 2 summarizes prior results in the probabilistic design of constant-coefficient controllers for linear systems. In Section 3, we present a general approach for probabilistic robust control of nonlinear systems. In Section 4, the proposed approach is applied to the flight control design for a NASA Langley hypersonic aircraft model and the design for the High Incidence Research Model is presented in Section 5; simulation results are presented for stability and performance robustness of the closedloop system.

\title{
2 Stochastic Analysis and Design for Linear, Time-Invariant Systems
}

\author{
Stochastic Robustness Analysis (SRA)
}

Stochastic stability theory provides a logical starting point, as satisfactory stability is often a necessary condition for satisfactory performance. A typical problem is to determine bounds on the parameter vector $\mathbf{p}$ of an unforced, continuous-time system $[22,24]$,

$$
\dot{\mathbf{x}}(t)=\mathbf{f}[\mathbf{p}(t), \mathbf{x}(t)], \mathbf{x} \in \mathbf{R}^{n}, \mathbf{x}(0)=\mathbf{x}_{0}, \mathbf{f} \in \mathbf{R}^{n}, \mathbf{p} \in \mathbf{R}^{l}
$$

where $\mathbf{x}$ is the dynamic state and $\mathbf{p}(t)$ is a random process, such that stability can be expected with a probability of one (or arbitrarily close to one). A corresponding linear control problem is to find a satisfactory control gain matrix $\mathbf{C}$ for the linear plant and control law,

$$
\begin{gathered}
\dot{\mathbf{x}}(t)=\mathbf{F}[\mathbf{p}(t), t] \mathbf{x}(t)+\mathbf{G}[\mathbf{p}(t), t] \mathbf{u}(t), \mathbf{u} \in \mathbf{R}^{m}, \mathbf{F} \in \mathbf{R}^{n \times n}, \mathbf{G} \in \mathbf{R}^{n \times m} \\
\mathbf{u}(t)=-\mathbf{C x}(t), \mathbf{C} \in \mathbf{R}^{m \times n}
\end{gathered}
$$

The system dynamics vector $\mathbf{f}(\cdot)$ becomes

$$
\mathbf{f}[\mathbf{p}(t), \mathbf{x}(t)]=\{\mathbf{F}[\mathbf{p}(t), t]-\mathbf{G}[\mathbf{p}(t), t] \mathbf{C}\} \mathbf{x}(t)
$$

and the uncertainty is contained in the varying values of $[\mathbf{F}(\cdot), \mathbf{G}(\cdot)]$. Probabilistic stability criteria have been developed using expectations of Lyapunov functions, and they require consideration of stochastic integrals and transformations [20, 59]. Analogous discrete-time problems are discussed in [25]. Given infinite (e.g., Gaussian) parameter distributions, the probability of instability is finite, and the escape (or exit) time may be of interest [11].

The principle focus of current robustness research is on ensembles of linear systems for which $\mathbf{p}$ is a random constant rather than a random process. For a particular parameter value $\mathbf{p}_{k}, \mathbf{F}_{\mathbf{p}_{k}}$ is uncertain but fixed. Deterministic stability criteria apply to each member of the ensemble. Because each dynamic system is linear and time-invariant, its stability is entirely determined by its eigenvalues, that is, the solutions $\lambda_{j}$ to the equation 


$$
\left|\lambda_{j} \mathbf{I}-\mathbf{F}^{T}\left(\mathbf{p}_{k}\right)\right|=0, j=1, \cdots, n
$$

Given a vector of the probability density functions of $\mathbf{p}, \operatorname{pr}(\mathbf{p})$, Eq. 5 provides an implicit transformation for computing the probability density functions, $\operatorname{pr}\left(\lambda_{j}\right)$, of the corresponding ensemble of eigenvalues $\lambda_{j}, j=1, \cdots, n$. An evaluation of the cumulative probability of (in)stability induced by $\operatorname{pr}(\mathbf{p})$ requires integration of the $\operatorname{pr}\left(\lambda_{j}\right)$ over the (right) left-half complex plane. Linear eigenvalue sensitivities, $\partial \lambda_{j} / \partial \mathbf{p}$, can be derived and applied for analytic evaluation of the integral $[27,40]$, and additional studies of eigenvalue and eigenvector sensitivities can be found in $[34,12,34,19,52,16,53]$.

Analytical solutions to this integral have limited utility for evaluating the probability of (in)stability. The most practical approach for evaluating the probability of (in)stability in the general case is to use numerical computation, as expanded below. Numerical evaluation of probabilities involves sampling of parameter probability distributions [39, 33] and computation of their consequences using either exhaustive sampling or "Monte Carlo" methods [8]. In the first case, all possible parameter combinations in a finite set are sampled, and the exact probability of hypothesis $\mathcal{H}$ is computed as

$$
\operatorname{Pr}(\mathcal{H})=N_{\mathcal{H}} / N_{\text {Total }}
$$

where $N_{\mathcal{H}}$ is the number of instances of $\mathcal{H}$, and $N_{\text {Total }}$ is the total number of trials. For the second method, each scalar parameter is represented by a random number generator, whose characteristics are shaped by the parameter's statistical description. There is no restriction on the shapes or correlations of probability distributions (i.e., they may be bounded, non-Gaussian, etc.), and parameters may have different distribution types. For a single trial, each element of $\mathbf{p}_{k}$ is generated, and the related hypothesis (in the current discussion, the stability or instability of the controlled system) is computed. The probability of a hypothesis is computed as before (Eq. 6), but there is uncertainty in the estimate, as discussed below.

For linear, time-invariant (LTI) systems, the probability of instability $P$ can be estimated from repeated eigenvalue calculation [54]. Given a system with $l$ parameters, each of which takes $w$ values with equal probability, $P$ can be calculated exactly from $w^{l}$ evaluations using exhaustive sampling (Eq. 6), with $N_{\mathcal{H}}$ equal to the number of unstable cases, and $N_{\text {Total }}$ equal to $w^{l}$. For Monte Carlo evaluation, the closed-loop eigenvalues, $\lambda_{j}$, are evaluated $N_{\text {Total }}$ times with each element of $\mathbf{p}_{k}, k=1, \cdots, N_{\text {Total }}$, specified by a random number generator whose individual outputs are shaped by $\operatorname{pr}(\mathbf{p})$. The probability-of(in)stability estimate becomes increasingly precise as $N_{\text {Total }}$ becomes large:

$$
\begin{aligned}
& \operatorname{Pr}(\text { stable })=\lim _{N_{\text {Total }} \rightarrow \infty} \frac{N\left(\sigma_{\max } \leq 0\right)}{N_{\text {Total }}} \\
& \operatorname{Pr}(\text { unstable })=P=1-\operatorname{Pr}(\text { stable })
\end{aligned}
$$

$N(\cdot)$ is the number of cases for which all elements of $\underline{\sigma}$, the vector of the real parts of the closed-loop eigenvalues $(\lambda=\sigma+j \omega)$, are less than or equal to 
zero, that is, for which $\sigma_{\max } \leq 0$, where $\sigma_{\max }$ is the maximum real eigenvalue component in $\underline{\sigma}$. For $N_{\text {Total }}<\infty$, the Monte Carlo evaluation is an estimate, $\hat{P}$, whose uncertainty is characterized by a confidence interval.

Because $P$ is a binomial variable (i.e., the outcome of each trial takes one of two values: stable or unstable), confidence intervals are calculated using the binomial test, where lower $(\mathcal{L})$ and upper $(\mathcal{U})$ intervals satisfy the following [10]:

$$
\begin{gathered}
\operatorname{Pr}\left(N_{U} \leq n-1\right)=\sum_{k=0}^{n-1}\left(N_{\text {Total }}, k\right) \mathcal{L}^{k}(1-\mathcal{L})^{N_{\text {Total }}-k}=1-\frac{\alpha}{2} \\
\operatorname{Pr}\left(N_{U} \leq n\right)=\sum_{k=0}^{n}\left(N_{\text {Total }}, k\right) \mathcal{U}^{k}(1-\mathcal{U})^{N_{\text {Total }}-k}=\frac{\alpha}{2}
\end{gathered}
$$

$N_{U}$ is the actual number of unstable cases after $N_{\text {Total }}$ evaluations $\left(N_{U}=\right.$ $\left.N_{\text {Total }} \hat{P}\right),\left(N_{\text {Total }}, k\right)$ is the binomial coefficient, $\frac{N_{\text {Total }} !}{k !\left(N_{\text {Total }}-k\right) !}$, and $(1-\alpha)$ is the confidence coefficient. Explicit approximations of the binomial test $[4,5]$ avoid an iterative solution of Eq. 9 and Eq. 10 for $(\mathcal{L}, \mathcal{U})$, and they are accurate to within $0.1 \%$ [54].

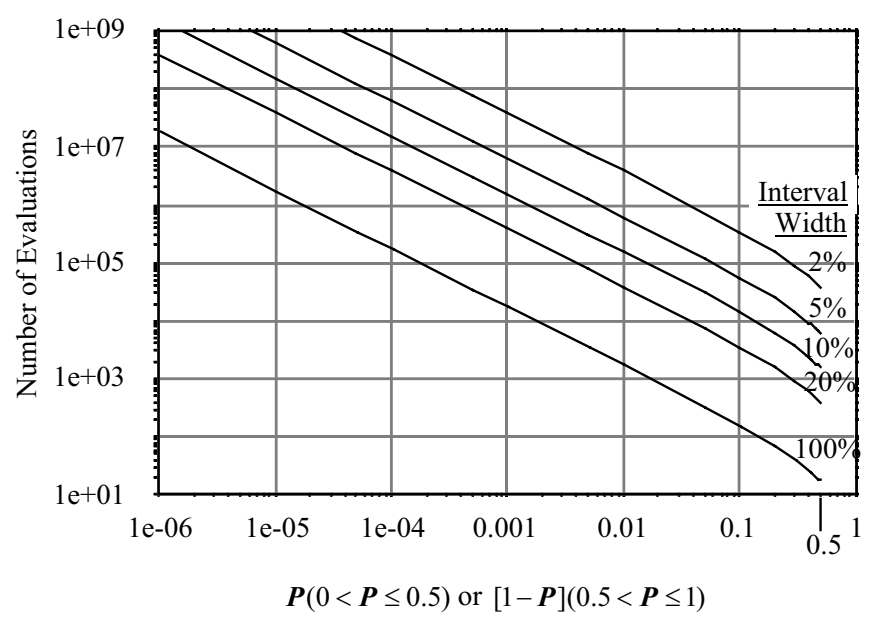

Fig. 1. Number of evaluations required to estimate a binomial probability distribution for given confidence interval widths and $95 \%$ confidence coefficient; interval width is given as percent of $P$ or $(1-P)$. (from [54]) 
The number of evaluations required to estimate a binomial probability distribution for specified interval widths and a 95\% confidence coefficient varies with the true $P$ (Fig. 1 [54]). For narrow intervals and small $P$, large numbers of evaluations are required; however, large percentage interval widths may be acceptable if $P$ is small.

The number of Monte Carlo evaluations needed to yield $\hat{P}$ with a given confidence level is independent of the number of uncertain parameters or their probability distributions. This result has broad implications for the robustness evaluation of complex systems. While exact or approximate exhaustive sampling may be useful when there are few parameters, Monte Carlo simulation has broad application for systems with large numbers of uncertain parameters.

\section{Stochastic Robustness Design(SRD)}

Design for stochastic robustness follows analysis by incorporating search. The simplest approach is to choose the best from an ensemble of controllers, without regard to the design algorithms employed for each controller. For example, given the Benchmark Control Problem [58], we could compare the probabilities of instability, $P_{i}$, excess control usage, $P_{u}$, and excess settling time, $P_{T_{s}}$, for the ten design solutions, selecting the one that appears most suitable. The relative importance of the three criteria must be known to make the selection, and the probability distributions of the uncertain parameters that induce them should be well motivated. Guidelines for comparing controller pairs are contained in [44].

Probabilistic synthesis of control systems is a natural adjunct to probabilistic analysis; the random or randomized search is a dual to Monte Carlo evaluation. Building on [51], random-search methods of finding control system gains are explored in $[6,60,55]$. There are similarities to directed searches that minimize multi-objective cost functions [48], to parameter-space methods [50, 1], and to fine-tuning of control gains by search [3]. A genetic algorithm - which performs randomized reproduction, crossover, and mutation on candidate control-gain strings - has been used to design controllers [23], while the stochastic robustness analysis is extended to control design using sequential line searches in [54, 42, 43, 44, 45, 46].

A typical design procedure has four steps: 1) define cost function of objective probabilities, 2) define controller structure, 3) perform stochastic robustness analysis of the closed-loop system, and 4) conduct numerical search to minimize the cost function.

As an example for Step 1, the quadratic cost function

$$
J=\alpha P_{i}^{2}+\beta P_{u}^{2}+\gamma P_{T_{s}}^{2}
$$

weights the squares of the probabilities to emphasize large values and deemphasize small values. $\alpha, \beta$, and $\gamma$ are scalar weights on the relative importance of instability, excess control usage, and excess settling time over the 
range of parameter uncertainty. $P_{i}, P_{u}$, and $P_{T_{s}}$ are in $(0,1)$. With an LQG controller, the control law and associated estimator for Step 2 are

$$
\begin{gathered}
u(t)=-C \hat{x} \\
\dot{\hat{x}}=F \hat{x}+G u+K(z-H \hat{x})
\end{gathered}
$$

and the weighting matrices for the LQG problem are chosen as the control design parameters. For a single-input/single-output compensator, the controller structure may simply be a transfer function whose numerator and denominator coefficients are the design parameters. In Step 3, an ensemble of trials is evaluated to compute the probability (Eq. 6) using the dynamic system of Eq. 2 with randomly generated parameter vectors, $\mathbf{p}$, and closed-loop control specified by Eq. 12 and Eq. 13. This Monte Carlo evaluation forms an "inner loop" for the minimization algorithm in Step 4. A genetic algorithm is used to minimize Eq. 11 through the choice of control design parameters. As an alternative, simulated annealing could be used for the optimization [35]. Execution time for this computationally intensive process can be decreased greatly through the use of parallel computation [47].

\section{Stochastic Robust Control of Nonlinear Systems}

The nonlinear control design is an extension of the probabilistic robust control of linear systems in Section 2. A combination of probabilistic robustness with feedback linearization is presented. First, we design a feedback linearization control law for the nominal system, then introduce parametric uncertainty and reformulate the problem in a probabilistic format. The control design parameters are searched to minimize a stochastic robustness cost function that is a weighted quadratic sum of probabilities of violating design specifications.

Consider a nonlinear system that has a nominal system as follows:

$$
\begin{aligned}
\dot{\mathbf{x}}=\mathbf{f}(\mathbf{x})+\mathbf{G}(\mathbf{x}) \mathbf{u}, \mathbf{G}(\mathbf{x})=\left[\mathbf{g}_{1}(\mathbf{x}) \mathbf{g}_{2}(\mathbf{x}) \cdots \mathbf{g}_{m}(\mathbf{x})\right] \\
\mathbf{y}=\mathbf{h}(\mathbf{x})
\end{aligned}
$$

where $\mathbf{f}$ and $\mathbf{g}_{j}(j=1,2, \cdots, m)$ are smooth vector fields on $\mathbf{R}^{n}$, and $\mathbf{h}$ is a smooth function mapping $\mathbf{R}^{n} \rightarrow \mathbf{R}^{m}$. If this nominal system is feedback linearizable, there exists a nonlinear coordinate transformation $\varsigma=T(\mathbf{x})$

$$
\left\{\begin{array}{l}
\varsigma_{1}^{i}=h_{i} \\
\varsigma_{2}^{i}=\frac{d h_{i}}{d t}=L_{\mathbf{f}} h_{i} \\
\vdots \\
\varsigma_{\lambda_{i}}^{i}=\frac{d^{\left(\lambda_{i}-1\right)} h_{i}}{d t}=L_{\mathbf{f}}^{\lambda_{i}-1} h_{i}
\end{array} \quad i=1,2, \cdots, m\right.
$$

such that the nominal system is transformed to a set of decoupled linear systems, 


$$
\left\{\begin{array}{l}
\dot{\zeta}_{1}^{i}=\varsigma_{2}^{i} \\
\dot{\zeta}_{2}^{i}=\varsigma_{3}^{i} \\
\vdots \\
\dot{\zeta}_{\lambda_{i}}^{i}=L_{\mathbf{f}}^{\lambda_{i}} h_{i}+\sum_{j=1}^{m} L_{\mathbf{g}_{j}}\left(L_{\mathbf{f}}^{\lambda_{i}-1} h_{i}\right) u_{j}=v_{i}
\end{array} \quad i=1,2, \cdots, m\right.
$$

where the Lie derivatives are defined as $L_{\mathbf{f}} h_{i}=\frac{\partial h_{i}(\mathbf{x})}{\partial \mathbf{x}} \mathbf{f}(\mathbf{x}), L_{\mathbf{f}}^{k} h_{i}=L_{\mathbf{f}}\left(L_{\mathbf{f}}^{k-1} h_{i}\right)$, and $L_{\mathbf{g}_{j}} h_{i}=\frac{\partial h_{i}(\mathbf{x})}{\partial \mathbf{x}} \mathbf{g}_{j}(\mathbf{x})$.

For the decoupled linear systems (17), the control law $\mathbf{v}=\left[\begin{array}{llll}v_{1} & v_{2} & \cdots & v_{m}\end{array}\right]^{T}$ could be designed using any existing technique, such as, a linear quadratic control that is parameterized in terms of weighting matrices $\mathbf{Q}$ and $\mathbf{R}$. By Eq. 17, the nonlinear control $u$ is calculated through the new control input $\mathbf{v}$ as

$$
\mathbf{u}=-\left[\mathbf{G}^{*}(\mathbf{x})\right]^{-1} \mathbf{f}^{*}(\mathbf{x})+\left[\mathbf{G}^{*}(\mathbf{x})\right]^{-1} \mathbf{v}
$$

where

$$
\begin{gathered}
\mathbf{f}^{*}(\mathbf{x})=\left(\begin{array}{c}
L_{\mathbf{f}}^{\lambda_{1}} h_{1} \\
L_{\mathbf{f}}^{\lambda_{2}} h_{2} \\
\vdots \\
L_{\mathbf{f}}^{\lambda_{m}} h_{m}
\end{array}\right) \\
\mathbf{G}^{*}(\mathbf{x})=\left(\begin{array}{cccc}
L_{\mathbf{g}_{1}} L_{\mathbf{f}}^{\lambda_{1}-1} h_{1} & L_{\mathbf{g}_{2}} L_{\mathbf{f}}^{\lambda_{1}-1} h_{1} & \cdots & L_{\mathbf{g}_{m}} L_{\mathbf{f}}^{\lambda_{1}-1} h_{1} \\
L_{\mathbf{g}_{1}} L_{\mathbf{f}}^{\lambda_{2}-1} h_{2} & L_{\mathbf{g}_{2}} L_{\mathbf{f}}^{\lambda_{2}-1} h_{2} & \cdots & L_{\mathbf{g}_{m}} L_{\mathbf{f}}^{\lambda_{2}-1} h_{2} \\
\cdots & \cdots & \cdots & \cdots \\
L_{\mathbf{g}_{1}} L_{\mathbf{f}}^{\lambda_{m}-1} h_{m} & L_{\mathbf{g}_{2}} L_{\mathbf{f}}^{\lambda_{m}-1} h_{m} & \cdots & L_{\mathbf{g}_{m}} L_{\mathbf{f}}^{\lambda_{m}-1} h_{m}
\end{array}\right)
\end{gathered}
$$

After the control design is derived for the nominal system, we consider the uncertain nonlinear vector fields $(\mathbf{f}(\mathbf{x}, \mathbf{q}), \mathbf{G}(\mathbf{x}, \mathbf{q}))$ subject to parametric uncertainty $\mathbf{q} \in \mathbf{Q}$. According to system design requirements, a set of robustness metrics is defined and a stochastic robustness cost function is formulated as a weighted quadratic sum of the probabilities of violating these robustness metrics. We use the parameterized control law of the nominal system as the controller structure for the system with uncertainties, and tune the control design parameters to minimize the stochastic robustness cost function. The inputto-state stability of the nominal closed-loop system is guaranteed, and the stability and other performance metrics of the uncertain system are evaluated by Monte Carlo simulation. As addressed in Section 2, the discrepancy between the Monte Carlo estimate and the true value results in apparent "noise" in the evaluation of the cost function. Furthermore, the cost function may be non-convex, having large plateaus and corners, so traditional gradient-based search algorithms can get stuck in local minima and not escape from large plateau areas. A series of randomized algorithms such as stochastic gradient methods, sequential line search, clustering algorithms, genetic algorithms and simulated annealing has been investigated [30, 35]. In this chapter, genetic algorithms are used to minimize the stochastic robustness cost function. 
In the following two sections, we illustrate the application of the above stochastic robust nonlinear control to two flight control examples: a NASA Langley hypersonic aircraft and the High Incidence Research Model. One of the major challenges in the design of flight control systems is model uncertainties and parameter variations in characterizing an aircraft and its operating environment. While many gains have been made in robust control theory over the past several decades, the gap between the new methods and conventional flight control design approaches has precluded their widespread use. The proposed stochastic robust control framework takes into account the engineering design requirements during the design phase, and it gives a direct answer to the likelihood that the design metrics are not satisfied.

\section{Stochastic Robust Control Design For a Hypersonic Aircraft Model}

System Model and Design Specifications

Consider the control of the longitudinal motion of a hypersonic aircraft cruising at a Mach number of 15 and at an altitude of 110,000 ft [49]. The dynamic equations are,

$$
\begin{gathered}
\dot{V}=\frac{T \cos \alpha-D}{m}-\frac{\mu \sin \gamma}{r^{2}} \\
\dot{\gamma}=\frac{L+T \sin \alpha}{m V}-\frac{\left(\mu-V^{2} r\right) \cos \gamma}{V r^{2}} \\
\dot{h}=V \sin \gamma \\
\dot{\alpha}=q-\dot{\gamma} \\
\dot{q}=M_{y y} / I_{y y}
\end{gathered}
$$

where

$$
\begin{gathered}
L=\frac{1}{2} \rho V^{2} S C_{L}(\alpha) \\
D=\frac{1}{2} \rho V^{2} S C_{D}(\alpha) \\
T=\frac{1}{2} \rho V^{2} S C_{T}(\delta T, \alpha) \\
M_{y y}=\frac{1}{2} \rho V^{2} S \bar{c}\left[C_{M}(\alpha)+C_{M}(\delta E)+C_{M}(q)\right] \\
r=h+R_{E}
\end{gathered}
$$

We have used relatively simple functions to fit the aerodynamic coefficients and air data around the nominal cruising condition. Twenty-eight inertial and aerodynamic parameters (identified in [56] due to the length of this chapter) are assumed to be uncertain. Each parameter is multiplied by an element 
of the uncertainty vector, $\nu$, that is assumed to follow a normal distribution with a mean of 1 and a standard deviation of 0.1 . At the trimmed cruise condition $(M=15, V=15,060 \mathrm{ft} / \mathrm{s}, h=110,000 \mathrm{ft}, \alpha=0.0315 \mathrm{rad}$, $\delta T=0.183, \delta E=-0.0066 \mathrm{rad}$, and $\left.T=4.6853 \times 10^{4} \mathrm{lbf}\right)$, a linearized model of the nominal open-loop dynamics has eigenvalues of $-0.8,0.687$, $-0.0001+0.0263 j$, and 0.0008 . The first two eigenvalues represent a statically unstable short-period mode; the complex pair of eigenvalues portrays a lightly damped phugoid mode, and the last real eigenvalue indicates a mildly unstable height mode. Consequently, cruising flight would be subject to attitude and height divergence that would require stabilizing feedback control.

Three aspects of flight control robustness are of concern in this design: stability, performance in velocity command response, and performance in altitude command response. The command responses are initiated at the trimmed condition. State histories of the aircraft's nonlinear response to the velocity and altitude commands are evaluated for stability and performance. Table 1 lists 39 stability and performance metrics that characterize the responses to a step velocity command change of $100 \mathrm{ft} / \mathrm{s}$ and a step altitude command change of $2,000 \mathrm{ft}$. The indicator functions with subscripts " $V$ " and " $h$ " denote the metrics for velocity and altitude command responses.

The cost function chosen to guide the design is a weighted quadratic sum of the 39 probabilities of design requirement violation:

$$
J=\sum_{j=1}^{39} w_{j} P_{j}^{2}
$$

As indicated in Table 1 , the stability weight $w_{1}$ is chosen as 10 , the weight for each more-demanding performance metric is selected as 1 , and the weight for each less-demanding performance metric is 0.1 .

\section{Controller Structure}

First we consider the nominal dynamics of the hypersonic aircraft with velocity and altitude commands:

$$
y_{\text {com }}=\left(\begin{array}{l}
V \\
h
\end{array}\right)
$$

Integral compensation is used to minimize the steady-state error of the command response; hence define

$$
V_{I}=\int_{0}^{t}\left(V(\tau)-V^{*}\right) d \tau, h_{I}=\int_{0}^{t}\left(h(\tau)-h^{*}\right) d \tau
$$

where $V^{*}$ and $h^{*}$ are the commanded values.

Dynamic extension is used to ensure that the vector relative degree is well defined; we assume that engine dynamics take a second-order form,

$$
\ddot{\delta T}=k_{1} \delta \dot{T}+k_{2} \delta T+k_{3} \delta T_{c o m}
$$


Table 1. Stability and performance metrics for a hypersonic aircraft

\begin{tabular}{|c|c|c|c|}
\hline Metric & $\begin{array}{c}\text { Weight } \\
\text { in J }\end{array}$ & $\begin{array}{c}\text { Indicator } \\
\text { Function }\end{array}$ & Design Requirement \\
\hline 1 & 10 & $I_{i}$ & Stability \\
\hline $2(3)$ & $0.1(1.0)$ & $I_{V, T_{s} 25}\left(I_{V, T_{s} 50}\right)$ & $10 \%$ settling time less than $25 \mathrm{~s}(50 \mathrm{~s})$ \\
\hline $4(5)$ & $0.1(1.0)$ & $I_{V, R 25}\left(I_{V, R 50}\right)$ & $90 \%$ rise time less than $25 \mathrm{~s}(50 \mathrm{~s})$ \\
\hline 6 & 0.1 & $I_{V, R e v}$ & No reversal of response in V before peaking \\
\hline $7(8)$ & $0.1(1.0)$ & $I_{V, D 5}\left(I_{V, D 10}\right)$ & $10 \%$ dwell time less than $5 \mathrm{~s}(10 \mathrm{~s})$ \\
\hline $9(10)$ & $0.1(1.0)$ & $I_{V, O S 10}\left(I_{V, O S 20}\right)$ & Overshoot less than $10 \%(20 \%)$ \\
\hline $11(12)$ & $0.1(1.0)$ & $I_{V, \Delta \alpha 0.5}\left(I_{V, \Delta \alpha 1}\right)$ & Max change in $\alpha$ less than $0.5^{\circ}\left(1^{\circ}\right)$ \\
\hline $13(14)$ & $0.1(1.0)$ & $I_{V, g 1}\left(I_{V, g 2}\right)$ & Max load factor less than $1 \mathrm{~g}(2 \mathrm{~g})$ \\
\hline $15(16)$ & $0.1(1.0)$ & $I_{V, \Delta h 0.25}\left(I_{V, \Delta h 0.5}\right)$ & Max change of h less than $0.25 \%(0.5 \%)$ \\
\hline $17(18)$ & $0.1(1.0)$ & $I_{V, \delta T 50}\left(I_{V, \delta T 100}\right)$ & Max change in thrust less than $50 \%(100 \%)$ \\
\hline $19(20)$ & $0.1(1.0)$ & $I_{V, \delta E 5}\left(I_{V, \delta E 10}\right)$ & Max change in $\delta E$ less than $5^{\circ}\left(10^{\circ}\right)$ \\
\hline $21(22)$ & $0.1(1.0)$ & $I_{h, T_{s} 50}\left(I_{h, T_{s} 100}\right)$ & $10 \%$ settling time less than $50 \mathrm{~s}(100 \mathrm{~s})$ \\
\hline $23(24)$ & $0.1(1.0)$ & $I_{h, R 50}\left(I_{h, R 100}\right)$ & $90 \%$ rise time less than $50 \mathrm{~s}(100 \mathrm{~s})$ \\
\hline 25 & 0.1 & $I_{h, R e v}$ & No reversal of response in h before peaking \\
\hline $26(27)$ & $0.1(1.0)$ & $I_{h, D 10}\left(I_{h, D 20}\right)$ & $10 \%$ dwell time less than $10 \mathrm{~s}(20 \mathrm{~s})$ \\
\hline $28(29)$ & $0.1(1.0)$ & $I_{h, O S 20}\left(I_{h, O S 40}\right)$ & Overshoot less than $20 \%(40 \%)$ \\
\hline $30(31)$ & $0.1(1.0)$ & $I_{h, \Delta \alpha 0.5}\left(I_{h, \Delta \alpha 1}\right)$ & Max change in $\alpha$ less than $0.5^{\circ}\left(1^{\circ}\right)$ \\
\hline $32(33)$ & $0.1(1.0)$ & $I_{h, g 1}\left(I_{h, g 2}\right)$ & Max load factor less than $1 \mathrm{~g}(2 \mathrm{~g})$ \\
\hline $34(35)$ & $0.1(1.0)$ & $I_{h, \Delta V 0.25}\left(I_{h, \Delta V 0.5}\right)$ & Max change of V less than $0.25 \%(0.5 \%)$ \\
\hline $36(37)$ & $0.1(1.0)$ & $I_{h, \delta T 50}\left(I_{h, \delta T 100}\right)$ & Max change in thrust less than $50 \%(100 \%)$ \\
\hline $38(39)$ & $0.1(1.0)$ & $I_{h, \delta E 5}\left(I_{h, \delta E 10}\right)$ & Max change in $\delta E$ less than $5^{\circ}\left(10^{\circ}\right)$ \\
\hline & & &
\end{tabular}

where choosing $k_{1}=k_{2}=0$ and $k_{3}=1$ provides a suitable model.

By augmenting the state variables as

$$
\mathbf{x}_{1}=\left(\begin{array}{c}
V_{I} \\
V \\
\gamma
\end{array}\right), \mathbf{x}_{2}=\left(\begin{array}{c}
\delta T \\
h_{I} \\
h \\
\alpha
\end{array}\right), \mathbf{x}_{3}=\left(\begin{array}{c}
\delta \dot{T} \\
q
\end{array}\right)
$$

and defining the control vector as

$$
\mathbf{u}=\left(\begin{array}{c}
\delta T_{\text {com }} \\
\delta E
\end{array}\right)
$$

the state equation can be put into a triangular form, i.e. it is feedback linearizable.

Using the notation

$$
\mathbf{z}^{T}=(V \gamma \alpha \delta T h)
$$

We have

$$
\left\{\begin{array}{l}
\dot{V}=\frac{T \cos \alpha-D}{m}-\frac{\mu \sin \gamma}{r^{2}} \\
\ddot{V}=\frac{1}{m} \omega_{1} \dot{\mathbf{z}} \\
V^{(3)}=\frac{1}{m}\left(\omega_{1} \ddot{\mathbf{z}}+\dot{\mathbf{z}}^{T} \Omega_{2} \dot{\mathbf{z}}\right)
\end{array}\right.
$$




$$
\left\{\begin{aligned}
\dot{h}=V & \sin \gamma \\
\ddot{h}=\dot{V} & \sin \gamma+V \dot{\gamma} \cos \gamma \\
h^{(3)}= & \ddot{V} \sin \gamma+2 \dot{V} \dot{\gamma} \cos \gamma-V \dot{\gamma}^{2} \sin \gamma+V \ddot{\gamma} \cos \gamma \\
h^{(4)}= & V^{(3)} \sin \gamma+3 \ddot{V} \dot{\gamma} \cos \gamma-3 \dot{V} \dot{\gamma}^{2} \sin \gamma+3 \ddot{V} \ddot{\gamma} \cos \gamma \\
& \quad-3 V \dot{\gamma} \ddot{\gamma} \sin \gamma-V \dot{\gamma}^{3} \cos \gamma+V \gamma^{(3)} \cos \gamma
\end{aligned}\right.
$$

where

$$
\left\{\begin{array}{l}
\ddot{\gamma}=\pi_{1} \dot{\mathbf{z}} \\
\gamma^{(3)}=\pi_{1} \ddot{\mathbf{z}}+\dot{\mathbf{z}}^{T} \Xi_{2} \dot{\mathbf{z}}
\end{array}\right.
$$

The vectors $\omega_{1}, \pi_{1}$ and matrices $\Omega_{2}, \Xi_{2}$ are omitted due to the length of the chapter; they can be found in [56].

By separating $\ddot{\alpha}$ and $\ddot{\delta T}$ into control-independent and control-dependent parts,

$$
\begin{gathered}
\ddot{\alpha}=\ddot{\alpha}_{0}+\ddot{\alpha}_{\delta E} \delta E \\
\ddot{\delta T}=\ddot{\delta T}_{0}+\delta \ddot{T}_{\text {com }} \delta T_{\text {com }}
\end{gathered}
$$

where $\ddot{\alpha}_{\delta E}$ represents the first derivatives of $\ddot{\alpha}$ with respect to $\delta E$, and $\ddot{\delta} \bar{T}_{\text {com }}$ represents the first derivative of $\ddot{\delta T}$ with respect to $\delta T_{\text {com }}, \ddot{\mathbf{z}}^{T}$ can be written as

$$
\begin{aligned}
\ddot{\mathbf{z}}^{T}= & (\ddot{V} \ddot{\gamma} \ddot{\alpha} \delta \ddot{T} \ddot{h}) \\
= & \left(\ddot{V} \ddot{\gamma} \ddot{\alpha}_{0} \ddot{\delta T_{0}} \ddot{h}\right) \\
& +\left(\delta T_{c o m} \delta E\right) \cdot\left(\begin{array}{ccccc}
0 & 0 & 0 & \ddot{\delta T}_{\text {com }} & 0 \\
0 & 0 & \ddot{\alpha}_{\delta E} & 0 & 0
\end{array}\right) \\
= & \ddot{\mathbf{z}}_{0}^{T}+\mathbf{u}^{T} \ddot{\mathbf{z}}_{\mathbf{u}}^{T}
\end{aligned}
$$

Therefore, by Eq. 38 and Eq. 39, we have

$$
\left(\begin{array}{c}
V^{(3)} \\
h^{(4)}
\end{array}\right)=\mathbf{f}^{*}(\mathbf{x})+\mathbf{G}^{*}(\mathbf{x}) \mathbf{u}
$$

with $\mathbf{f}^{*}$ and $\mathbf{G}^{*}$ as

$$
\begin{aligned}
\mathbf{f}^{*} & =\left(\begin{array}{c}
\frac{1}{m} \omega_{1} \ddot{\mathbf{z}}_{0}+\frac{1}{m} \dot{\mathbf{z}}^{T} \Omega_{2} \dot{\mathbf{z}} \\
3 \ddot{V} \dot{\gamma} \cos \gamma-3 \dot{V} \dot{\gamma}^{2} \sin \gamma+3 \dot{V} \ddot{\gamma} \cos \gamma-3 V \dot{\gamma} \ddot{\gamma} \sin \gamma-V \dot{\gamma}^{3} \cos \gamma \\
+\left(\frac{1}{m} \omega_{1} \ddot{\mathbf{z}}_{0}+\frac{1}{m} \dot{\mathbf{z}}^{T} \Omega_{2} \dot{\mathbf{z}}\right) \sin \gamma+V \cos \gamma\left(\pi_{1} \ddot{\mathbf{z}}_{0}+\dot{\mathbf{z}}^{T} \Pi_{2} \dot{\mathbf{z}}\right)
\end{array}\right) \\
\mathbf{G}^{*} & =\left(\begin{array}{cc}
\frac{T_{\delta T} \cos \alpha}{m} \delta T_{\operatorname{com}} & \frac{T_{\alpha} \cos \alpha-T \sin \alpha-D_{\alpha}}{m} \ddot{\alpha}_{\delta E} \\
\frac{T_{\delta T} \sin (\alpha+\gamma)}{m} \delta T_{\operatorname{com}} & \frac{T \cos (\alpha+\gamma)+T_{\alpha} \sin (\alpha+\gamma)+L_{\alpha} \cos \gamma-D_{\alpha} \sin \gamma}{m} \ddot{\alpha}_{\delta E}
\end{array}\right)
\end{aligned}
$$

The determinant of $\mathbf{G}^{*}$ is calculated as

$$
\operatorname{det}\left(\mathbf{G}^{*}\right)=\frac{T_{\delta T} \ddot{\delta T}_{c o m} \ddot{\alpha}_{\delta E}}{m^{2}} \cos \gamma\left(T+L_{\alpha} \cos \alpha+D_{\alpha} \sin \alpha\right)
$$

where $L_{\alpha}, D_{\alpha}$ and $T_{\alpha}$ denote the partial derivatives of $L, D$, and $T$ with respect to the angle of attach $\alpha ; T_{\delta T}$ denotes the partial derivative of $T$ with 
respect to the throttle setting $\delta T$. The nonsingular condition for $\mathbf{G}^{*}$ can be represented as

$$
\operatorname{det}\left(\mathbf{G}^{*}\right) \neq 0 \Leftrightarrow\left(T+L_{\alpha} \cos \alpha+D_{\alpha} \sin \alpha\right) \cos \gamma \neq 0
$$

Therefore, $\mathbf{G}^{*}$ is nonsingular unless the flight path is vertical or $\left(T+L_{\alpha} \cos \alpha+\right.$ $\left.D_{\alpha} \sin \alpha\right)=0$.

By assuming desired command-rates as zero, and using Eq. 38 and Eq. 39, we define a nonlinear coordinate transformation, $\xi=T_{1}\left(\mathbf{x}, V^{*}\right)$ and $\eta=T_{2}\left(\mathbf{x}, h^{*}\right)$, as

$$
\left\{\begin{array} { l } 
{ \xi _ { 1 } = \int _ { 0 } ^ { t } ( V ( \tau ) - V ^ { * } ) d \tau } \\
{ \xi _ { 2 } = V - V ^ { * } } \\
{ \xi _ { 3 } = \dot { V } } \\
{ \xi _ { 4 } = \ddot { V } }
\end{array} \quad \left\{\begin{array}{l}
\eta_{1}=\int_{0}^{t}\left(h(\tau)-h^{*}\right) d \tau \\
\eta_{2}=h-h^{*} \\
\eta_{3}=\dot{h} \\
\eta_{4}=\ddot{h} \\
\eta_{5}=h^{(3)}
\end{array}\right.\right.
$$

This results in decoupled subsystems

$$
\begin{aligned}
& \dot{\xi}=\mathbf{A}_{1} \xi+\mathbf{b}_{1} v_{1} \\
& \dot{\eta}=\mathbf{A}_{2} \eta+\mathbf{b}_{2} v_{2}
\end{aligned}
$$

where

$$
\begin{aligned}
& \mathbf{A}_{1}=\left(\begin{array}{llll}
0 & 1 & 0 & 0 \\
0 & 0 & 1 & 0 \\
0 & 0 & 0 & 1 \\
0 & 0 & 0 & 0
\end{array}\right), \mathbf{b}_{1}=\left(\begin{array}{l}
0 \\
0 \\
0 \\
1
\end{array}\right) \\
& \mathbf{A}_{2}=\left(\begin{array}{lllll}
0 & 1 & 0 & 0 & 0 \\
0 & 0 & 1 & 0 & 0 \\
0 & 0 & 0 & 1 & 0 \\
0 & 0 & 0 & 0 & 1 \\
0 & 0 & 0 & 0 & 0
\end{array}\right), \mathbf{b}_{2}=\left(\begin{array}{l}
0 \\
0 \\
0 \\
0 \\
1
\end{array}\right)
\end{aligned}
$$

For the transformed linear systems, Eq. 50 and Eq. 51, we design the new inputs $v_{1}$ and $v_{2}$ as linear-quadratic control laws. Considering intermediate objective functions

$$
J_{1}=\int_{0}^{\infty}\left(\xi^{T} \mathbf{Q}_{1} \xi+r_{1} v_{1}^{2}\right) d t
$$

and

$$
J_{2}=\int_{0}^{\infty}\left(\eta^{T} \mathbf{Q}_{2} \eta+r_{2} v_{2}^{2}\right) d t
$$

the new input $v_{1}$ is derived by minimizing $J_{1}$ subject to Eq. 50 : 


$$
v_{1}=-r_{1}^{-1} \mathbf{b}_{1}^{T} \mathbf{P}_{1} \xi
$$

$\mathbf{P}_{1}$ is the positive-definite solution to the algebraic Riccati equation with design parameters $\mathbf{Q}_{1}$ and $r_{1}$,

$$
\mathbf{A}_{1}^{T} \mathbf{P}_{1}+\mathbf{P}_{1} \mathbf{A}_{1}-r_{1}^{-1} \mathbf{P}_{1} \mathbf{b}_{1} \mathbf{b}_{1}^{T} \mathbf{P}_{1}+\mathbf{Q}_{1}=0,\left(\mathbf{Q}_{1}, r_{1}>0\right)
$$

Similarly, minimizing $J_{2}$ subject to Eq. 51 gives

$$
v_{2}=-r_{2}^{-1} \mathbf{b}_{2}^{T} \mathbf{P}_{2} \eta
$$

$\mathbf{P}_{2}$ is the positive-definite solution to the Riccati equation with design parameters $\mathbf{Q}_{2}$ and $r_{2}$,

$$
\mathbf{A}_{2}^{T} \mathbf{P}_{2}+\mathbf{P}_{2} \mathbf{A}_{2}-r_{2}^{-1} \mathbf{P}_{2} \mathbf{b}_{2} \mathbf{b}_{2}^{T} \mathbf{P}_{2}+\mathbf{Q}_{2}=0,\left(\mathbf{Q}_{2}, r_{2}>0\right)
$$

The nonlinear control law $\mathbf{u}$ is obtained by inserting $\mathbf{v}=\left(v_{1} v_{2}\right)^{T}$ into Eq. 18,

$$
\mathbf{u}=-\left(\mathbf{G}^{*}(\mathbf{x})\right)^{-1} \mathbf{f}^{*}(\mathbf{x})+\left(\mathbf{G}^{*} \mathbf{x}\right)^{-1}\left(\begin{array}{c}
-r_{1}^{-1} \mathbf{b}_{1}^{T} \mathbf{P}_{1} \xi \\
-r_{2}^{-1} \mathbf{b}_{2}^{T} \mathbf{P}_{2} \eta
\end{array}\right)
$$

In the next step we consider the system robustness subject to the variations of the uncertain aerodynamic parameters. Appropriate $\mathbf{Q}_{1}, r_{1}, \mathbf{Q}_{2}$, and $r_{2}$ in the intermediate objective functions are found by minimizing the stochastic robustness cost function (Eq. 31). For simplicity, we choose the design parameters $\mathbf{Q}_{1}=\operatorname{diag}\left\{q_{1}, q_{2}, q_{3}, q_{4}\right\}$ and $\mathbf{Q}_{2}=\operatorname{diag}\left\{q_{5}, q_{6}, q_{7}, q_{8}, q_{9}\right\}$, and the design parameter vector is

$$
\mathbf{d}=\left(q_{1}, q_{2}, \cdots, q_{9}, r_{1}, r_{2}\right)
$$

Satisfactory values of the eleven design parameters in Eq. 61 are computed by applying a genetic algorithm to Monte Carlo estimates of the stochastic robustness cost function (31).

Stochastic Robustness Analysis of the Design Result

The design parameter vector, Eq. 61, found by a genetic algorithm after 20 generations, is given as

$$
\begin{aligned}
\mathbf{d}= & \left(8.54 \times 10^{-6}, 0.34,0.86,47.93,1.1 \times 10^{-11}, 2.35 \times 10^{-3},\right. \\
& 0.52,220.6,57.12,0.89,1.05)
\end{aligned}
$$

The performance for the nominal closed-loop system is shown in Fig. 2. Figure 2(a) shows the response due to a $100 \mathrm{ft} / \mathrm{s}$ step-velocity command from the trimmed condition $(V=15,060 \mathrm{ft} / \mathrm{s}, h=110,000 \mathrm{ft})$. The velocity converges to the command value in $30 \mathrm{~s}$ with little change in altitude and with a change of angle of attack of less than $0.06^{0}$. We note that the use of thrust is unrealistically high, as there are limits to the thrust available. Nevertheless, the example illustrates the effectiveness of the design approach for the specified criteria. Figure 2(b) shows the velocity, altitude change, and control input 


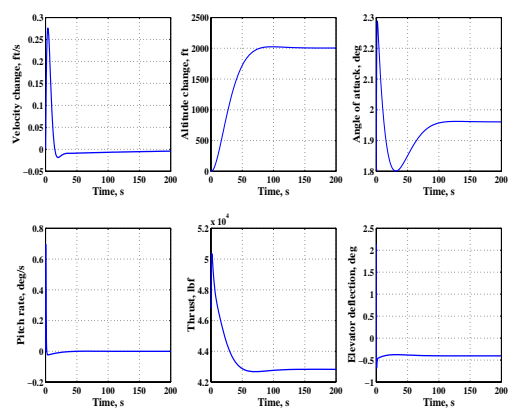

(a)
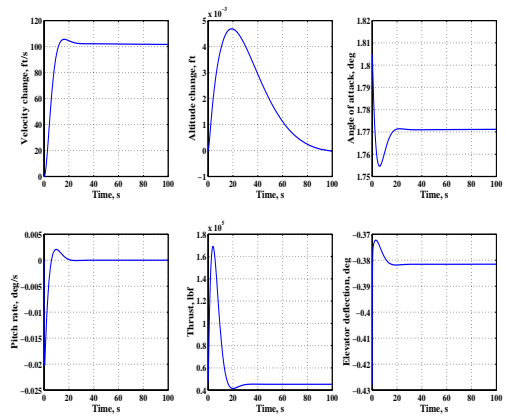

(b)

Fig. 2. (a) Response to a $100 \mathrm{ft} / \mathrm{s}$ step-velocity command; (b) Response to a 2,000 ft step-altitude command.

time histories to a $2,000 \mathrm{ft}$ step-altitude command. The altitude converges to the command value in $75 \mathrm{~s}$, with a change of angle of attack of less than $0.5^{0}$. Figure 2 demonstrates that the nominal system has good performance.

Figure 3 shows the robustness comparison of the current feedback linearization control (nonlinear dynamic inversion NDI) in (62) to a linear quadratic (LQ) design [32] based on two thousand Monte Carlo evaluations. The nonlinear design (NDI) has a cost of $J=1.23$, while the linear design LQ has a cost of $J=1.72$. The closed-loop probability of instability of the nonlinear design equals zero with a $95 \%$ confidence interval of $(0,0.0018)$; it has $5 \%$ to $56 \%$ lower probability of exceeding settling time than the LQ design (Metrics 2-3 and 21-22) and $15 \%$ to $80 \%$ lower probability of exceeding rise time (Metrics 7-8 and 26-27). The nonlinear design has also reduced the probability of exceeding load factor by more than $80 \%$ compared to the LQ design (Metrics 13-14 and 32-33).

The NDI has larger probability of exceeding control effort corresponding to Metric $18, I_{V, \delta T 100}$, and Metric $36, I_{h, \delta T 50}$, due to the possibility that nonlinear dynamic inversion may cancel some useful nonlinearities. Furthermore, in Eq. 54 and Eq. 55, the weights $r_{1}$ and $r_{2}$ penalize large inputs $v_{1}$ and $v_{2}$ instead of penalizing thrust directly as in LQ. We can see that NDI performs better than LQ in Metric $19(20), I_{V, \delta E 5}\left(I_{V, \delta E 10}\right)$, and Metric $38(39), I_{h, \delta E 5}$ $\left(I_{h, \delta E 10}\right)$. The robustness profiles can be adjusted by changing the weights in the robustness cost function. For example, trade-offs between using less thrust and accepting longer rise time, or putting heavy weight on $P_{h, T_{s}}$ to decrease the probability of exceeding settling-time can be easily examined. 


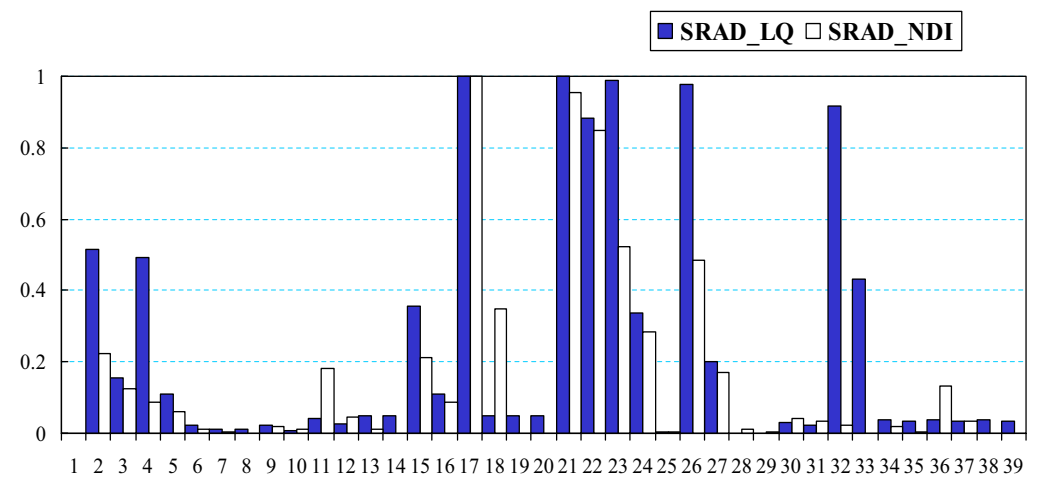

Fig. 3. Comparison of the robustness profiles of the stochastic robust control based on linear quadratic regulator structure (LQ), and nonlinear dynamic inversion structure (NDI).

\section{Stochastic Robust Control Design for the High Incidence Research Model}

The HIRM aircraft configuration has canard and tailplane control surfaces plus an elongated nose. The mathematical model uses aerodynamic data obtained from wind tunnel and flight tests of an unpowered, scaled drop model. Engine, sensor, and actuator models have been added to the mathematical model to create a representative nonlinear simulation of a twin-engine modern fighter. The aircraft is basically stable both longitudinally and laterally, although there are some combinations of angle of attack and control surface deflections that cause the aircraft to be unstable. Reference [36] described in detail the six-degree-of-freedom nonlinear High Incidence Research Model including nonlinear actuator and sensor models. We first present the dynamic equations of motion for a general aircraft, and then address the aerodynamics for the HIRM problem.

\subsection{System Model and Design Metrics}

Equations of Motion

The dynamic equations of motion for an aircraft in a combined wind and body axes are written as follows [14]: 


$$
\begin{gathered}
\dot{V}=\frac{F_{w x}}{m}-g \sin \gamma \\
\dot{\alpha}=q-\frac{q w}{\cos \beta}-p \cos \alpha \tan \beta-r \sin \alpha \tan \beta \\
\dot{\beta}=r_{w}+p \sin \alpha-r \cos \alpha \\
\dot{\gamma}=q_{w} \cos \varphi-r_{w} \sin \varphi \\
\dot{\varphi}=p_{w}+\left(q_{w} \sin \varphi+r_{w} \cos \varphi\right) \tan \gamma \\
\dot{\psi}=\frac{q_{w} \sin \varphi+r_{w} \cos \varphi}{\cos \gamma} \\
\dot{q}=\frac{1}{I_{y y}}\left[\begin{array}{c}
\left.\mathcal{M}+I_{x z}\left(r^{2}-p^{2}\right)+\left(I_{z z}-I_{x x}\right) r p\right] \\
\dot{\dot{r}}
\end{array}\right)=\left(\begin{array}{cc}
I_{x x} & -I_{x z} \\
-I_{x z} & I_{z z}
\end{array}\right)\left(\begin{array}{c}
\mathcal{L}+I_{x z} p q+\left(I_{y y}-I_{z z} q r\right. \\
\mathcal{N}-I_{x z} q r+\left(I_{x x}-I_{y y} p q\right.
\end{array}\right)
\end{gathered}
$$

with

$$
\begin{gathered}
q_{w}=-\frac{F_{w z}}{m V}-\frac{g}{V} \cos \gamma \cos \varphi \\
r_{w}=\frac{F_{w y}}{m V}+\frac{g}{V} \cos \gamma \sin \varphi \\
p_{w}=p \cos \alpha \cos \beta+(q-\dot{\alpha}) \sin \beta+r \sin \alpha \cos \beta \\
\left(\begin{array}{c}
\mathcal{L} \\
M \\
N
\end{array}\right)=\left(\begin{array}{c}
L_{A} \\
M_{A} \\
N_{A}
\end{array}\right)+\left(\begin{array}{c}
L_{T} \\
M_{T} \\
N_{T}
\end{array}\right) \\
\left(\begin{array}{c}
F_{w x} \\
F_{w y} \\
F_{w z}
\end{array}\right)=-\left(\begin{array}{c}
D \\
S \\
L
\end{array}\right)+\left(\begin{array}{c}
T_{w x} \\
T_{w y} \\
T_{w z}
\end{array}\right)
\end{gathered}
$$

Here, $V=$ flight path velocity, $\alpha=$ angle of attack, $\beta=$ sideslip angle, $(\gamma, \varphi, \psi)$ $=$ wind-axis Euler angles, $(p, q, r)=$ body-axis angular rates, $\left(p_{w}, q_{w}, r_{w}\right)=$ wind-axis angular rates; $(L, M, N)=$ body-axis total rolling, pitching, and yawing moments, $\left(L_{A}, M_{A}, N_{A}\right)=$ body-axis aerodynamic moments, $\left(L_{T}, M_{T}, N_{T}\right)$ $=$ body-axis moments due to engine thrust, $\left(F_{w x}, F_{w y}, F_{w z}\right)=$ wind-axis total forces, $(D, S, L)=$ drag, side, and lift force in wind axis, and $\left(T_{w x}, T_{w y}, T_{w z}\right)$ $=$ wind-axis thrust.

The transformation matrix from body axis to wind axis is defined as:

$$
L_{W B}=\left(\begin{array}{ccc}
\cos \alpha \cos \beta & \sin \beta & \sin \alpha \cos \beta \\
-\cos \alpha \sin \beta & \cos \beta & -\sin \alpha \sin \beta \\
-\sin \alpha & 0 & \cos \alpha
\end{array}\right)
$$

The Mach number $M$ is defined as $M=\frac{V}{a}$. 


\section{Aerodynamics}

Body-axis aerodynamic forces and moments, $\left(F_{x A}, F_{y A}, F_{z A}\right)$ and $\left(L_{A}, M_{A}, N_{A}\right)$, are represented in terms of the non-dimensional aerodynamic coefficients $\left(C_{X}, C_{Y}, C_{Z}\right)$ and $\left(C_{l}, C_{m}, C_{n}\right)$ as follows:

$$
\left\{\begin{array}{c}
F_{x}=\frac{1}{2} \rho V^{2} S C_{X} \\
F_{y}=-\frac{1}{2} \rho V^{2} S C_{Y} \\
F_{z}=\frac{1}{2} \rho V^{2} S C_{Z}
\end{array},\left\{\begin{array}{c}
\mathcal{L}_{A}=\frac{1}{2} \rho V^{2} S b C_{l} \\
\mathcal{M}_{A}=\frac{1}{2} \rho V^{2} S \bar{c} C_{m} \\
\mathcal{N}_{A}=\frac{1}{2} \rho V^{2} S b C n
\end{array}\right.\right.
$$

where $\rho$ denotes the air density, $S$ denotes the aircraft's wing planform area, $b$ denotes the span, and $\bar{c}$ denotes the mean aerodynamic chord. The aerodynamic force and moment coefficients are highly nonlinear functions of angle of attack $\alpha$, sideslip angle $\beta$, airspeed $V$, angular rates $p, q, r$, and control deflections (symmetrical and differential taileron deflections $\delta_{T S}$ and $\delta_{T D}$, symmetrical and differential canard deflections $\delta_{C S}$ and $\delta_{C D}$, rudder deflection $\delta_{R}$, and engine throttle $\delta_{T H}$ ). Each component of the aerodynamic force and moment coefficients is represented by a look-up table. Details on the high-fidelity model can be found in [36].

\section{Pilot Commands}

The pilot commands should control the responses as follows: lateral stick deflection commands velocity-vector roll rate $p_{w c}$, which is a roll performed at constant angle of attack and zero sideslip; longitudinal stick deflection commands pitch rate $q_{c}$; rudder pedal deflection commands sideslip angle $\beta_{c}$; throttle lever deflection commands velocity-vector air speed $V_{c}$, which represents a step command from its trim value $V_{\text {trim }}$.

\section{Design Envelope}

The flight envelope that is specified by the GARTEUR/HIRM competition and used in comparison has Mach number within $(0.15,0.5)$, angle of attack $\left(-10^{\circ}, 30^{\circ}\right)$, sideslip angle $\left(-10^{\circ}, 10^{\circ}\right)$, and altitude $(100 \mathrm{ft}, 20000 \mathrm{ft})$.

\section{Modelling Errors}

The control system should be robust to the errors in the aerodynamic moment derivatives and to the biases in the total moment coefficients. The variation of $C_{m_{w}}$ is within $(-0.001,0.001)$, variation of $C_{l_{v}}$ is within $(-0.01,0.01)$, and the variation of $C_{n_{v}}$ is within $(0.002,0.002)$. The variations of $C_{m_{q}}, C_{l_{p}}, C_{n_{r}}, C_{l_{r}}$, $C_{n_{p}}, C_{m_{T S}}, C_{m_{C S}}, C_{l_{T D}}, C_{l_{C D}}, C_{l_{R U D D E R}}, C_{n_{T D}}, C_{n_{C D}}$, and $C_{n_{R U D D E R}}$ are within $(-10 \%, 10 \%)$ of the derivative's trim values. Though these uncertainties are proposed for linear analysis in [36], we include these aerodynamic-momentderivative uncertainties in the assessment of nonlinear time responses. We assume that the uncertainties take uniform distributions in the designated ranges. 


\section{Formulation of the Robustness Metrics}

In Table 2, we formulate robustness metrics in keeping with performance requirements in the assessments of a set of required maneuvers. All the robustness metrics are evaluated by Monte Carlo simulations with random number generators providing possible values of the uncertain aerodynamic parameters. It is assumed that the uncertain parameters take uniform distributions in the designated ranges.

Table 2. Formulation of robustness metrics

\begin{tabular}{|c|c|c|c|}
\hline Metric & $\begin{array}{l}\text { Weight } \\
\text { in } \mathrm{J}\end{array}$ & $\begin{array}{l}\text { Indicator } \\
\text { Function }\end{array}$ & Design Requirement \\
\hline 1 & 10 & $I_{i}$ & Stability at all flight conditions \\
\hline \multicolumn{4}{|c|}{ Pitch rate command response } \\
\hline 2,3 & 1.0 & $\begin{array}{l}I_{3 q_{-} q T_{s}} \\
I_{5 q_{-} q T_{s}}\end{array}$ & $\begin{array}{l}10 \% \text { settling time less than } 2 \mathrm{~s} \text { for pitch rate } \\
\text { command response at } \mathrm{M}=0.3 \text { and } 0.5\end{array}$ \\
\hline $4,5,6$ & 0.1 & \begin{tabular}{l|}
$I_{2 q \_a \max }$ \\
$I_{3 q \_a \max }$ \\
$I_{5 q \_a \max }$ \\
\end{tabular} & $\begin{array}{l}\quad-10^{\circ}<\alpha<30^{\circ} \text { for pitch rate } \\
\text { demand response at } \mathrm{M}=0.2,0.3 \text {, and } 0.5\end{array}$ \\
\hline $7,8,9$ & 0.1 & \begin{tabular}{l|}
$I_{2 q_{-} z \max }$ \\
$I_{3 q_{-} z \max }$ \\
$I_{5 q_{-} z \max }$ \\
\end{tabular} & $\begin{array}{l}-3 g<a_{n z}<7 g \text { for pitch rate } \\
\text { demand response at } \mathrm{M}=0.2,0.3, \text { and } 0.5\end{array}$ \\
\hline \multicolumn{4}{|c|}{ Velocity command response } \\
\hline 10 & 1.0 & $I_{3 V-V T_{s}}$ & $\begin{array}{l}10 \% \text { settling time less than } 15 \mathrm{~s} \\
\text { for velocity response at } \mathrm{M}=0.3\end{array}$ \\
\hline 11 & 0.1 & $I_{3 V_{-} q t}$ & $\begin{array}{l}\text { Pitch rate transient less than } 10^{\circ} / \mathrm{s} \\
\text { for velocity response at } \mathrm{M}=0.3\end{array}$ \\
\hline \multicolumn{4}{|c|}{ Sideslip command response } \\
\hline $12,13,14$ & 1.0 & $\begin{array}{l}I_{2 b} \\
I_{3 b} \\
I_{5 b}\end{array}$ & $\begin{array}{c}\text { Sideslip command response } \\
\text { lies within specified boundaries } \\
\text { at } \mathrm{M}=0.2,0.3 \text {, and } 0.5\end{array}$ \\
\hline \multicolumn{4}{|c|}{ Roll rate command response } \\
\hline 15,16 & 1.0 & $\begin{array}{l}I_{3 p_{-} p T_{s}} \\
I_{5 p_{-} p T_{s}}\end{array}$ & $\begin{array}{l}10 \% \text { settling time less than } 2 \mathrm{~s} \text { for } \\
\text { roll rate command response at } \mathrm{M}=0.3 \text { and } 0.5\end{array}$ \\
\hline 17,18 & 0.1 & $\begin{array}{l}I_{3 p_{-} q t} \\
I_{5 p_{-} q t}\end{array}$ & $\begin{array}{l}\text { Pitch rate transient less than } 5^{\circ} / \mathrm{s} \\
\text { for roll rate command response at } \mathrm{M}=0.3 \text { and } 0.5\end{array}$ \\
\hline
\end{tabular}

In Table 2 , the first indicator function, $I_{i}$, measures system stability. The system stability is evaluated in terms of the simulation of nonlinear time response. If all of the step command responses listed in Table 2 do not have finite escape time, we specify $I_{i}=0$; otherwise, $I_{i}=1$. Indicator functions 2-9 characterize the nonlinear time responses to step pitch-rate commands at different flight conditions. The angles of attack during pitch-rate commands should be within the specified limits with maximum overshoot less than $5^{\circ}$. The normal acceleration should be within the specified limits with maximum 
overshoot less than $0.5 \mathrm{~g}$. The settling time requirement is not specified for the pitch-rate response at $M=0.2$ because the necessity of an angle-ofattack limiter could cause transients of the pitch rate. Indicator functions 10-11 characterize the step velocity command response at $M=0.3$. Indicator functions 12-14 are for sideslip-angle command responses. The step response to sideslip command should lie within some specified boundaries [36]. Indicator functions 15-18 illustrate the requirements for roll-rate command responses.

The stochastic robustness cost function chosen to guide the design is a weighted quadratic sum of the eighteen probabilities of design metric violations:

$$
J=\sum_{j=1}^{18} w_{j} P_{j}^{2}
$$

The weight for each probability is given in Table 2 .

\subsection{Controller Structure}

The design of the controller structure is based on nonlinear dynamic inversion. It is possible to separate system dynamics into two time scales if one subset of the state components (referred to as "fast dynamics") is known to evolve in a much faster time scale than the other subset (referred to as "slow dynamics"). The inversion performed here is based on the assumption that the dynamics of angular rates are faster than those of angles of attack and sideslip. The design of controller structure is separated into two steps relating to the slow and fast dynamics.

For the slow dynamics, commanded angular rates are derived through either direct pilot inputs or the inversion of the force equations. The engine throttle position is derived through the inversion of the velocity dynamics. The values of yaw rate and engine throttle are obtained in terms of design parameters that characterize desired dynamics of sideslip angle and velocity. For the fast dynamics, control surface deflections are derived explicitly through the inversion of a first-order differentiation of angular velocities. They are defined in terms of design parameters that characterize desired dynamics of angular rates. The procedure of this two-time-scale nonlinear dynamic inversion is illustrated in Fig. 4.

\section{Slow Dynamics}

Design of the controller for slow dynamics shown in Fig. 4 deals with force equations and the kinematics' equation for velocity-vector roll rate. The purpose of this inversion is to derive command angular rates $\left(p_{c}, r_{c}\right)$ for the fast dynamics from the pilot commands $\left(p_{w c}, \beta_{c}\right)$, and to derive engine throttle position $\delta_{T H}$ from the pilot command velocity $V_{c}$.

First, we rewrite the equations for $\dot{\alpha}, \dot{\beta}, \dot{V}$, and $p_{w}$ in appropriate forms. The wind-axis thrust induced by the two engines is derived from the body-axis thrust: 


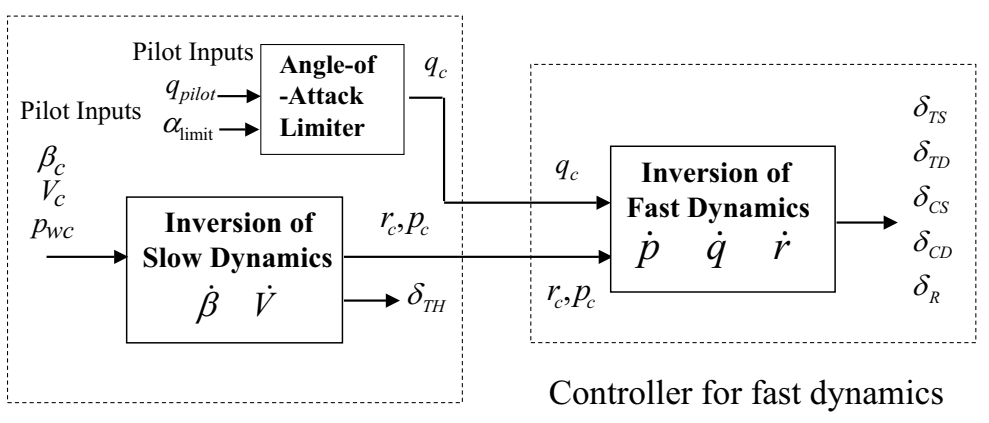

Controller for slow dynamics

Fig. 4. Controller structure designed using two-time-scale nonlinear dynamic inversion.

$$
\left(\begin{array}{c}
T_{x w} \\
T_{y w} \\
T_{z w}
\end{array}\right)=L_{W B}\left(\begin{array}{c}
T_{x} \\
T_{y} \\
T_{z}
\end{array}\right)=L_{W B}\left(\begin{array}{c}
2 F_{E} \\
0 \\
0
\end{array}\right)=\left(\begin{array}{c}
2 F_{E} \cos \alpha \cos \beta \\
-2 F_{E} \cos \alpha \sin \beta \\
-2 F_{E} \sin \alpha
\end{array}\right)
$$

By Eq. 79, Eq. 75 becomes

$$
\left(\begin{array}{c}
F_{w x} \\
F_{w y} \\
F_{w z}
\end{array}\right)=\left(\begin{array}{c}
-D+2 F_{E} \cos \alpha \cos \beta \\
-S-2 F_{E} \cos \alpha \sin \beta \\
-L-2 F_{E} \sin \alpha
\end{array}\right)
$$

We define wind axis load factors as

$$
\begin{gathered}
n_{w x}=\frac{F_{w x}}{m g}=\frac{-D+2 F_{E} \cos \alpha \cos \beta}{m g} \\
n_{w y}=\frac{F_{w y}}{m g}=\frac{-S-2 F_{E} \cos \alpha \sin \beta}{m g} \\
n_{w z}=\frac{F_{w z}}{m g}=\frac{-L-2 F_{E} \sin \alpha}{m g}
\end{gathered}
$$

Equation 71 and Eq. 72 are rewritten in terms of wind-axis load factor as,

$$
q_{w}=-\frac{g}{V}\left(\cos \gamma \cos \varphi+n_{w z}\right)
$$




$$
r_{w}=\frac{g}{V}\left(\cos \gamma \cos \varphi+n_{w y}\right)
$$

By setting $\dot{\alpha}$ and $\beta$ to zero in Eq. 73, we have

$$
p_{w c}=(p \cos \alpha+r \sin \alpha)
$$

With Eq. 85 and Eq. 86, Eq. 65 becomes

$$
\dot{\beta}=-\frac{r}{\cos \alpha}+p_{w c} \tan \alpha+\frac{g}{V}\left(n_{w y}+\cos \gamma \cos \varphi\right) \cos \alpha
$$

By Eq. 75, Eq. 63 becomes

$$
\dot{V}=\frac{2 F_{E} \cos \alpha \cos \beta-D}{m}-g \sin \gamma
$$

Next, we formulate the state and control inputs for the slow dynamics. Integral compensation is used to minimize steady-state error of the command response. Therefore, we define new state variables

$$
\begin{gathered}
V_{I}=\int_{0}^{t}\left[V(\tau)-\left(V_{\text {trim }}+V_{c}\right)\right] d \tau \\
\beta_{I}=\int_{0}^{t}\left(\beta(\tau)-\beta_{c}\right) d \tau
\end{gathered}
$$

The corresponding augmented state vector for slow dynamics is defined as:

$$
\mathbf{x}_{s}=\left(V_{I} V \beta_{I} \beta\right)^{T}
$$

The dynamic model for $\mathbf{x}_{s}$ is

$$
\left(\begin{array}{c}
\dot{V}_{I} \\
\dot{V} \\
\dot{\beta}_{I} \\
\dot{\beta}
\end{array}\right)=\left(\begin{array}{c}
V-\left(V_{\text {trim }}+V_{c}\right) \\
-2 \xi_{V} \omega_{V}\left[V-\left(V_{\text {trim }}+V_{c}\right)\right]-\omega_{V}^{2} V_{I} \\
\beta-\beta_{c} \\
-2 \xi_{\beta} \omega_{\beta}\left(\beta-\beta_{c}\right)-\omega_{\beta}^{2} \beta_{I}
\end{array}\right)
$$

where $\xi_{V}, \omega_{V}, \xi_{\beta}$, and $\omega_{\beta}$ are design parameters. $\xi_{V}$ and $\omega_{V}$ denote the desired damping ratio and frequency for velocity dynamics, while $\xi_{\beta}$ and $\omega_{\beta}$ denote the desired damping ratio and frequency for the dynamics of sideslip angle.

The control vector for slow dynamics consists of the thrust of each engine $F_{E}$ and the commanded yaw rate $r_{c}$ for the fast dynamics. Utilizing Eq. 87, Eq. 88 and Eq. 92, we derive the control vector $\mathbf{u}_{s}=\left(F_{E} r_{c}\right)^{T}$,

$$
\begin{gathered}
F_{E}=\frac{m}{2 \cos \alpha \cos \beta}\left\{\frac{D}{m}+g \sin \gamma-2 \xi_{V} \omega_{V}\left[V-\left(V_{\text {trim }}+V_{c}\right)\right]-\omega_{V}^{2} V_{I}\right\} \\
r_{c}=p_{w c} \cos \beta \sin \alpha+\frac{g}{V}\left(n_{w y}+\cos \gamma \sin \varphi\right) \cos \alpha \\
+\left[2 \xi_{\beta} \omega_{\beta}\left(\beta-\beta_{c}\right)+\omega_{\beta}^{2} \beta_{I}\right] \cos \alpha
\end{gathered}
$$


By Eq. 86 and Eq. 94, we derive the commanded roll rate $p_{c}$ for the fast dynamics as

$$
\begin{aligned}
p_{c}= & p_{w c} \cos \beta \cos \alpha-\frac{g}{V}\left(n_{w y}+\cos \gamma \sin \varphi\right) \sin \alpha \\
& +\left[-2 \xi_{\beta} \omega_{\beta}\left(\beta-\beta_{c}\right)-\omega_{\beta}^{2} \beta_{I}\right] \sin \alpha
\end{aligned}
$$

In terms of the engine model in [36], the throttle position is

$$
\delta_{T H}= \begin{cases}\frac{F_{E} \frac{\rho_{0}}{\rho}-F_{I D L E}}{F_{M D}-F_{I D L E}}, & F_{E} \frac{\rho_{0}}{\rho}<F_{M D} \\ 1+\frac{F_{E} \frac{\rho_{0}}{\rho}-F_{M D}}{F_{M R}-F_{M D}}, & F_{M D} \leq F_{E} \frac{\rho_{0}}{\rho} \leq F_{M R}\end{cases}
$$

with $F_{E}$ given by Eq. 93. $F_{I D L E}, F_{M D}$, and $F_{M R}$ denote the idle thrust, maximum dry thrust, and maximum reheat thrust for the engine.

The computation of $r_{c}, p_{c}$, and $F_{E}$ is conducted as follows. Through the transformation from body axes to wind axes $L_{W B}$, the wind-axis load factor $n_{w y}$ in Eq. 94 and Eq. 95 is calculated from the body-axis accelerations $a_{n x}$, $a_{n y}$, and $a_{n z}$, which are measured variables. Also through $L_{W B}$, drag $D$ in Eq. 93 is calculated from body-axis aerodynamic forces $F_{x A}, F_{y A}$, and $F_{z A}$, which are computed in terms of the aerodynamic force coefficients $C_{X}, C_{Y}$ and $C_{Z}$ by Eq. 77. The calculation of $C_{X}, C_{Y}$ and $C_{Z}$ depends on the values of control surface deflections, which are unknown and are computed in the phase of fast dynamics. In this chapter, the values of control surface deflections of the previous time iteration are used in computing aerodynamic force coefficients $C_{X}, C_{Y}$, and $C_{Z}$.

An angle-of-attack limiter is important because the commanded pitch rate $q_{c}$, which is an input for the fast dynamics, should be chosen as the minimum of the pilot-commanded pitch rate $q_{\text {pilot }}$ and the pitch rate $q_{\text {limit }}$ that would induce the maximum allowable angle of attack $\alpha_{\text {limit }}$,

$$
q_{c}=\min \left(q_{\text {pilot }}, q_{\text {limit }}\right)
$$

In terms of Eq. 64 and Eq. 84, $q_{\text {limit }}$ is derived as

$$
\begin{aligned}
q_{\text {limit }}= & (p \cos \alpha+r \sin \alpha) \tan \beta \\
& -\frac{g}{V} \frac{n_{w z}+\cos \gamma \cos \varphi}{\cos \beta}+\dot{\alpha}_{L I M}
\end{aligned}
$$

where the maximum allowable angle-of-attack rate, $\dot{\alpha}_{L I M}$, is calculated from

$$
\dot{\alpha}_{L I M}=-\omega_{\alpha}\left(\alpha-\alpha_{\text {limit }}\right)
$$

where $\omega_{\alpha}$ is a design parameter that denotes the bandwidth of the angle-ofattack control loop; $\alpha$ is the current angle of attack. The limit of angle of attack $\alpha_{\text {limit }}$ equals $30^{\circ}$. 


\section{Fast Dynamics}

Design of the controller corresponding to the fast dynamics in Fig. 4 consists of the inversion of the moment equations. The purpose of this inversion is to derive a vector of control surface deflections for a given set of commanded angular rates $p_{c}, q_{c}$ and $r_{c}$.

Integral compensation minimizes the steady-state error of the pitch rate command response; thus we define a new state variable,

$$
q_{I}=\int_{0}^{t}\left(q(\tau)-q_{c}\right) d \tau
$$

The state vector for the fast dynamics is

$$
\mathbf{x}_{f}=\left(\begin{array}{llll}
p & r & q_{I} & q
\end{array}\right)^{T}
$$

The dynamic model for angular rates is

$$
\begin{gathered}
\dot{p}=-\omega_{p}\left(p-p_{c}\right) \\
\dot{r}=-\omega_{r}\left(r-r_{c}\right) \\
\dot{q}=-2 \xi_{q} \omega_{q}\left(q-q_{c}\right)-\omega_{q}^{2} q_{I}
\end{gathered}
$$

where $\xi_{q}, \omega_{q}, \omega_{p}$, and $\omega_{r}$ are design parameters. $\xi_{q}$ and $\omega_{q}$ denote the desired damping ratio and frequency for the dynamics of pitch rate while $\omega_{p}$ and $\omega_{r}$ denote the desired bandwidths for $p$ and $r$.

The vector of control inputs for the fast dynamics consists of control surface deflections of the taileron, canard, and rudder:

$$
\mathbf{u}_{f}=\left(\delta_{T S} \delta_{T D} \delta_{C S} \delta_{C D} \delta_{R}\right)^{T}
$$

From Eq. 69, Eq. 70, Eq. 74, and Eq. 102, Eq. 103, Eq. 104, we have

$$
\begin{aligned}
\left(\begin{array}{c}
\mathcal{L}_{A} \\
\mathcal{N}_{A} \\
\mathcal{M}_{A}
\end{array}\right)= & -\left(\begin{array}{c}
\mathcal{L}_{T} \\
\mathcal{N}_{T} \\
\mathcal{M}_{T}
\end{array}\right)+\left(\begin{array}{c}
-I_{x z} p q+\left(I_{z z}-I_{y y}\right) q r \\
I_{x z} q r+\left(I_{y y}-I_{x x}\right) p q \\
\left(I_{x x}-I_{z z}\right) r p+I_{x z}\left(p^{2}-r^{2}\right)
\end{array}\right) \\
& +\left(\begin{array}{ccc}
I_{x x} & -I_{x z} & 0 \\
-I_{x z} & I_{z z} & 0 \\
0 & 0 & I_{y y}
\end{array}\right)\left(\begin{array}{c}
-\omega_{p}\left(p-p_{c}\right) \\
-\omega_{r}\left(r-r_{c}\right) \\
-2 \xi_{q} \omega_{q}\left(q-q_{c}\right)-\omega_{q}^{2} q_{I}
\end{array}\right)
\end{aligned}
$$

Note that the aerodynamic moments $\mathcal{L}_{A}, \mathcal{N}_{A}$, and $\mathcal{M}_{A}$ are nonlinear functions of the control surface deflections $\mathbf{u}_{f}$; the inverse mappings of these nonlinear functions have to be calculated in order to derive the control surface deflections $\mathbf{u}_{f}$. For simplicity of calculation, we approximate the aerodynamic moments by their first-order expansions with respect to control surface deflections around the values of control surface deflections at the previous time iteration: 


$$
\left(\begin{array}{c}
\mathcal{L}_{A} \\
\mathcal{N}_{A} \\
\mathcal{M}_{A}
\end{array}\right) \cong \frac{1}{2} \rho V^{2} \bar{S} \Lambda\left(\mathbf{u}_{f}-\mathbf{u}_{f}^{*}\right)+\frac{1}{2} \rho V^{2} \bar{S} \Upsilon
$$

Matrices $\Lambda$ and $\Upsilon$, which are functions of the control surface deflections at the previous time iteration $\mathbf{u}_{f}^{*}$, are given in [56].

Note that in Eq. 107, we have more unknown variables $\left(\mathbf{u}_{f}\right.$ consists of five control surface deflections) than equations (three equations); hence, the solution of $\mathbf{u}_{f}$ is not unique. We derive the control $\mathbf{u}_{f}$ in terms of $\Lambda^{\sharp}$, which is the pseudo-inverse of matrix $\Lambda$,

$$
\mathbf{u}_{f}=\mathbf{u}_{f}^{*}+\Lambda^{\sharp}\left\{\frac{1}{\frac{1}{2} \rho V^{2} \bar{S}}\left(\begin{array}{c}
\mathcal{L}_{A} \\
\mathcal{N}_{A} \\
\mathcal{M}_{A}
\end{array}\right)-\Upsilon\right\}
$$

where $\left(\mathcal{L}_{A} \mathcal{N}_{A} \mathcal{M}_{A}\right)^{T}$ is given by Eq. 106. The (right) pseudo-inverse operation used here corresponds to a minimization of the normalized control surface deflections.

We concatenate the control design parameters in Eq. 92 and Eq. 102, Eq. 103, Eq. 104 into a single design vector as

$$
\mathbf{d}=\left(\xi_{V} \omega_{V} \xi_{\beta} \omega_{\beta} \omega_{\alpha} \xi_{q} \omega_{q} \omega_{p} \omega_{r}\right)^{T}
$$

\subsection{Control Design Results}

The design parameter vector in Eq. 109 for our robust HIRM controller is found by using a genetic algorithm as follows:

$$
\mathbf{d}=\left(\begin{array}{llll}
0.419 & 1.0462 .8720 .4894 .9831 .4483 .0634 .0232 .663
\end{array}\right)^{T}
$$

The performance of the nominal closed-loop system is illustrated by a set of maneuvers in Fig. 5 and Fig. 6; the time responses for other maneuvers can be found in [28]. The figures show histories of the command variables and state variables of interest. The command values of pitch-rate, velocityvector-roll-rate, airspeed, and sideslip angle are plotted using dashed lines. The response to command is good in all cases. For the $5^{\circ} / \mathrm{s}$ pitch rate commanded response at $M=0.2$, Fig. 5 (a) shows angle of attack being limited to the maximum value, $30^{\circ}$. The pitch-rate transient that occurs at $t=5 \mathrm{sec}$ is due to this limiting. With the increase of the pitch attitude, the gravitational force component from the mass of the aircraft induces an additional force in the wind $\mathrm{x}$-axis that results in the variation of the airspeed. The thrust is increased to compensate for the change in attitude. For the $70 \% / \mathrm{s}$ roll rate commanded response at $M=0.5$, Fig. 5 (b) shows good performance. The roll rate follows the command input quite well, with $10 \%$ settling time less than 2 seconds. The coupling to sideslip angle is less than $1.5^{\circ}$, and the coupling to pitch rate is less than $1^{o} / s$. 


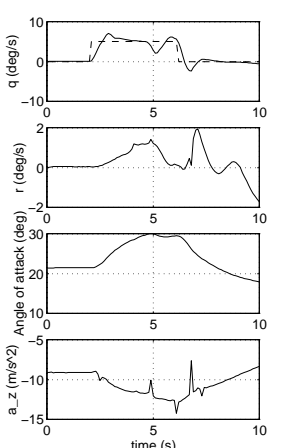

(a)

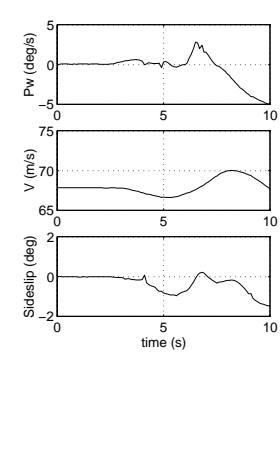

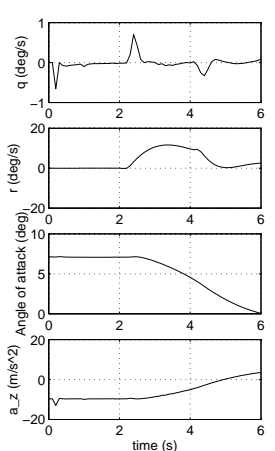

(b)

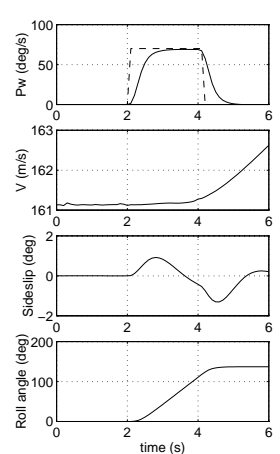

Fig. 5. (a) Pitch rate command response at $M=0.2$; (b) Roll rate command response at $\mathrm{M}=0.5$.

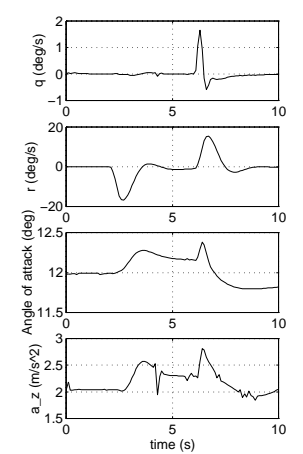

(a)
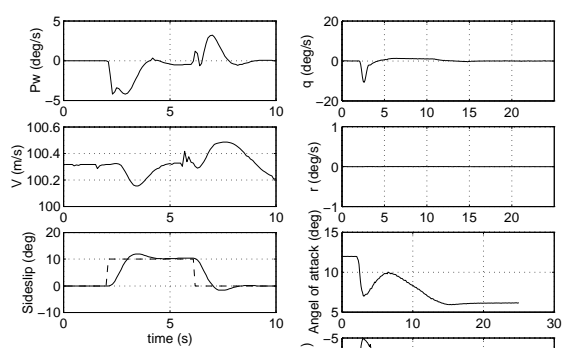

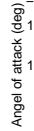
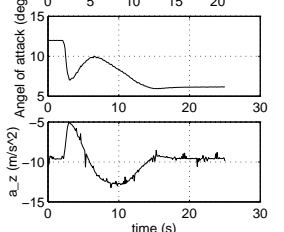

(b)
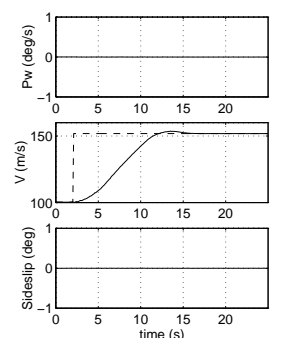

Fig. 6. (a) Sideslip angle command response at $M=0.3$; (b) Velocity command response at $\mathrm{M}=0.3$.

Figure 6(a) illustrates the responses due to a $10 \% / s$ step command on sideslip angle at $M=0.3$. The time history of the sideslip angle is well within the specified boundaries. It follows the command input with $10 \%$ settling time of less than 2 seconds. The couplings into roll and pitch rate are low. Figure 6(b) shows a $51.48 \mathrm{~m} / \mathrm{s}(100 \mathrm{kn})$ step on velocity commanded response at $M=0.3$. The $10 \%$ settling time is less than 15 seconds, and the overshoot is within $3 \%$. The pitch rate transient is low and returns to zero quickly. The engine is fully used for the rapid speed command change. The maximum throttle position is attained. The noise in the time history of normal acceleration $a_{z}$ is due to the relatively high bandwidth of the velocity. The control system shows good performance for the entire flight envelope including extreme flight conditions such as $30^{\circ}$ angle of attack. It is demonstrated that the controller has strong ability to account for significant nonlinearities. 


\subsection{Comparison of Present Design with Controllers Developed for GARTEUR Competition}

A set of control designs has been presented for the HIRM control challenges in the GARTEUR competition [28]. They include controllers based on linear-quadratic (LQ) methods [2], $H_{\infty}$ loop-shaping approaches [38], $\mu$ synthesis [17, 29], nonlinear dynamic inversion combined with linear-quadratic regulator (NDI/LQ) [13], and robust inverse dynamics estimation approaches (RIDE) [37]. The first three design approaches are linear techniques. Gain scheduling of linear feedback gains was utilized to cover the whole operating envelope of the aircraft. Reference [13] used a two-level controller structure consisting of a nonlinear-dynamic-inversion feedforward controller and a linear-quadratic feedback controller. In [13], the simulations for the nonlinear time responses were performed with the nonlinear-dynamic-inversion feedforward controller alone, without the linear-quadratic correction. Reference [37] combined dynamic inversion with proportional and integral feedback loops. Robustness issues were not directly taken into account in [37].

It is difficult to compare the present controller in this chapter against the designs presented in the GARTEUR competition because they were not intended to minimize the probabilities of metric violations subjected to expected parameter variations, as is the present design. In the evaluation software provided by GARTEUR, a single set of values of uncertain parameters is used to test a control system's robustness (deterministic characterization of uncertainties). Furthermore, very limited simulation results were presented for each design. Nevertheless, we provide a comparison of the present controller with the earlier designs based on the available information.

\section{Performance in Nominal Control Responses}

For each design in the GARTEUR competition, maneuver simulations are offered only at some of the flight conditions. There are no results shown for the commanded time responses in the presence of parameter uncertainties. A comparison of the performance of nominal time responses for a set of maneuvers between the present controller and previous designs is given in Table 3.

In Table 3, " $T_{s}$ " denotes a $10 \%$ settling time for a command response. " $T_{w}$ " represents the overshoot wash-out time for the angle of attack above its limiting value. A two-second wash-out time is required. We use "-" to denote unavailable results. Inadequate performances of each control design are highlighted.

The linear-quadratic design has quite good performance except that there is a slight excess of overshoot in the velocity command response, compared to the desired specification of less than $3 \%$. The $H_{\infty}$ loop-shaping controller has excess wash-out time for angle-of-attack overshoot above $30^{\circ}$ in the pitchrate command response, excess steady-state offsets of the roll-rate command response, and excess overshoot in the velocity command response. The first $\mu$-synthesis design has large steady-state offsets for the pitch-rate command 
response and excess settling time for the roll-rate command response. The second $\mu$-synthesis design has very good performance, except the settling time is longer than the required two seconds for the pitch rate command response. The NDI/LQ design has large overshoot in the velocity command response; otherwise, it demonstrates excellent nominal performance. The RIDE design has no overshoot in velocity, but there are slight steady-state offsets, and it has relatively long settling time for the sideslip-angle command response. Compared to previous designs in the GARTEUR competition, the controller presented in this chapter shows less overshoot in the velocity command response, faster response in all the maneuvers, and more accurate tracking of the commands without steady state offsets.

Table 3. Comparison of nominal performance for a set of controllers ("o.s." denotes overshoot).

\begin{tabular}{|c|c|c|c|c|c|c|c|}
\hline & LQ & $H \infty$ & $\mu-1$ & $\mu-2$ & NDI/LQ & RIDE & Present \\
\hline \multicolumn{8}{|c|}{ Pitch rate command responses } \\
\hline $\mathrm{M}=0.2$ & - & $\begin{array}{c}\alpha>30^{\circ} \\
\mathrm{w} / 1.5^{\circ} \\
\text { o.s. } \\
T_{w}>2 s\end{array}$ & - & - & $\begin{array}{c}\alpha \leq 30^{\circ} \\
\text { w/o } \\
\text { o.s. }\end{array}$ & - & $\begin{array}{c}\alpha \leq 30^{\circ} \\
\text { w/o } \\
\text { o.s. }\end{array}$ \\
\hline $\mathrm{M}=0.3$ & $T_{s}<2 s$ & - & $T_{s}<2 s$ & $T_{s}>2 s$ & - & - & $T_{s}<2 s$ \\
\hline $\mathrm{M}=0.4$ & - & - & - & - & - & $\begin{array}{l}\alpha \leq 30^{\circ} \\
\text { w/o o.s. }\end{array}$ & - \\
\hline $\mathrm{M}=0.5$ & - & - & $\begin{array}{l}\text { q offset } \\
=14 \%\end{array}$ & - & - & - & $T_{s}<2 s$ \\
\hline \multicolumn{8}{|c|}{ Roll rate command responses } \\
\hline $\mathrm{M}=0.3$ & $\begin{array}{c}T_{s}<2 s \\
|q|<7^{o} / s \\
|\beta|<4^{o}\end{array}$ & $\begin{array}{c}|q|<5^{\circ} / s \\
|\beta|<1.2^{\circ} \\
\text { p offset } \\
=20 \%\end{array}$ & $\begin{array}{c}T_{s}>2 s \\
|q|<8^{\circ} / s \\
|\beta|<0.7^{\circ}\end{array}$ & - & - & $\begin{array}{c}- \\
|\beta|<2^{o}\end{array}$ & $\begin{array}{c}T_{s}<2 s \\
|q|<4^{o} / s\end{array}$ \\
\hline $\mathrm{M}=0.4$ & - & - & - & - & $\begin{array}{c}T_{s}<2 s \\
|q|<2^{o} / s\end{array}$ & $\begin{array}{c}T_{s}<2 s \\
|q|<3^{\circ} / s\end{array}$ & - \\
\hline $\mathrm{M}=0.5$ & - & - & - & $\begin{array}{c}T_{s}<2 s \\
|q|<1^{o} / s\end{array}$ & - & - & $\begin{array}{c}T_{s}<2 s \\
|q|<1^{o} / s\end{array}$ \\
\hline \multicolumn{8}{|c|}{ Sideslip angle command response } \\
\hline $\mathrm{M}=0.2$ & - & - & - & - & - & - & $T_{s}<2 s$ \\
\hline $\mathrm{M}=0.3$ & $T_{s}<2 s$ & - & - & - & - & - & $T_{s}<2 s$ \\
\hline $\mathrm{M}=0.4$ & - & - & - & - & $T_{s}<2 s$ & $T_{s}>2 s$ & - \\
\hline $\mathrm{M}=0.5$ & - & $T_{s}<2 s$ & - & - & - & - & $T_{s}<2 s$ \\
\hline \multicolumn{8}{|c|}{ Velocity command response } \\
\hline $\mathrm{M}=0.3$ & $6.7 \%$ o.s. & $8 \%$ o.s. & - & - & $\begin{array}{l}20 \% \text { o.s. } \\
6 \% \text { offset }\end{array}$ & $\begin{array}{c}\text { w/o o.s. } \\
4.5 \% \text { offset }\end{array}$ & $<3 \%$ o.s. \\
\hline
\end{tabular}




\section{Performance Robustness in Linear Frequency Responses with Parametric} Uncertainties

In the GARTEUR competition, the evaluation software analyzes linear frequency responses of controllers in the presence of parametric uncertainties in moment derivatives. Linear frequency specifications have less value for our nonlinear control law; therefore, we do not include them in the formulation of our cost function. Nevertheless, our controller is evaluated against linear frequency requirements specified in the GARTEUR competition for comparison with earlier designs. The open-loop Nichols plot of the frequency response between each actuator demand $u$ and the corresponding error signal $e$ should avoid a gain-phase exclusion region. The evaluation is made in the presence of parametric uncertainties as: $C_{m_{v}}=-0.001, C_{l_{v}}=-0.01, C_{n_{v}}=-0.002, C_{l_{r}}$, $C_{n_{p}}=10 \%$, and $C_{m_{q}}, C_{l_{p}}, C_{n_{r}}, C_{m_{T S}}, C_{m_{C S}}, C_{l_{T D}}, C_{l_{C D}}, C_{l_{R U D D E R}}, C_{n_{T D}}$, $C_{n_{C D}}, C_{n_{R U D D E R}}=-10 \%$.

Open-loop Nichols plots for the present controller with parametric uncertainties are plotted in Fig. 7 for a flight condition at Mach $0.24,20,000 \mathrm{ft}$ altitude, $28.9^{\circ}$ angle of attack, and zero sideslip angle. This flight condition represents an edge of the flight envelope, which is likely to cause stability and actuator-limiting problems. Figure 7 shows that the frequency responses for all of the six control loops (differential and symmetrical taileron loops; differential and symmetrical canard loops; rudder loop, and thrust loop) avoid the specified gain-phase exclusion zone. In comparison to existing designs, for each of the controllers except the NDI/LQ and $H_{\infty}$ (lack of robustness information) in the GARTEUR competition, one loop's linear frequency response cannot satisfy the robustness criteria. We conclude that the nonlinear controller of this chapter shows better performance robustness than the earlier designs as portrayed by linear frequency analysis.

Linear frequency analysis is inadequate for evaluating nonlinear dynamic systems and nonlinear control laws. Furthermore, a single set uncertainty that is not proved to be the worst case for the parametric uncertainties is not enough to quantify system robustness. Two thousand Monte Carlo evaluation of the present design with controller parameters in Eq. 110 give the probabilistic robustness profile in Fig. 8. The confidence interval for each probability is not shown due to space limitations and can be found in [56]. In the Monte Carlo simulations, random number generators with uniform distributions provide the possible values of the system uncertain parameters. The design cost equals 1.14. The control system has a zero probability of instability (Metric $1)$ with a $95 \%$ confidence interval of $(0,0.0018)$. For the pitch-rate command response at $M=0.2$, adding the angle-of-attack limiter causes transients in pitch rate; therefore, the settling-time specification is not evaluated. The pitch-rate command response at $M=0.3$ is quite good, with low probability of excess settling time (Metric $2, I_{3 q_{-} q T_{s}}$ ). The probability of violating settlingtime condition at $M=0.5$ (Metric $3, I_{5 q_{-} q T_{s}}$ ) is more than double the probability at $M=0.3$. It is within expectation because $M=0.2$ and 0.5 repre- 


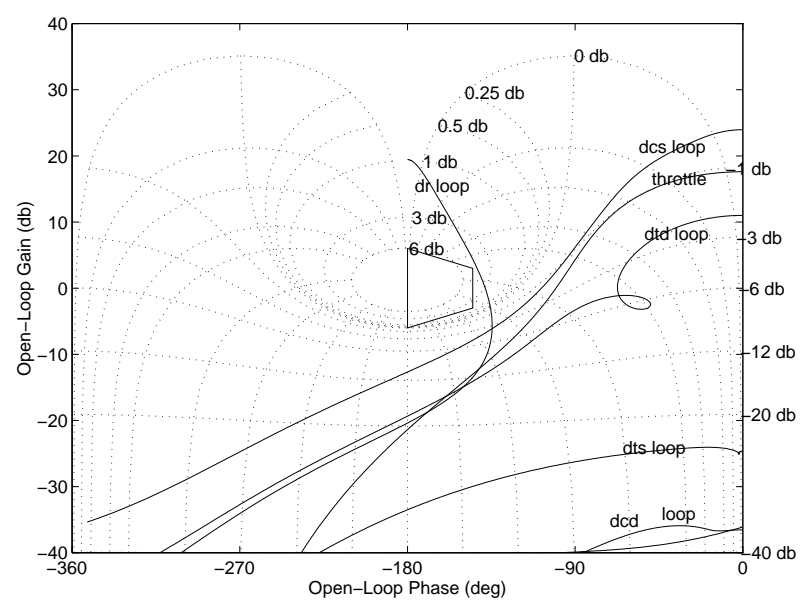

Fig. 7. Open loop Nichols plots of the present controller in the presence of parametric uncertainties with a flight condition at $\mathrm{M}=0.24$. The trapezoid denotes the gain-phase exclusion region.

sent edge-of-the-envelope flight conditions, and $M=0.3$ represents a nominal flight condition within the envelope. The probabilities of exceeding angle-ofattack and normal-acceleration limits in pitch-rate command responses equal zero (Metric 4-9) for all flight conditions with $95 \%$ confidence intervals of $(0,0.0018)$. Figure 8 shows that the probability of exceeding settling time for the velocity-command response is relatively high (Metric $10, I_{3 V_{-} V T_{s}}$ ), which is caused by the uncertainties in yawing moments and derivatives. The probability of pitch-rate coupling for velocity command is low (Metric 11, $I_{3 V_{-} q t}$ ). The performance robustness for sideslip-angle command responses is fine for each flight condition. The probabilities of violating settling time condition are about 20\% (Metrics 12-14). For roll-rate command responses, there are about $30 \%$ probability of excess settling time (Metrics 15-16) and less than 20\% probability of pitch-rate coupling for all flight conditions (Metrics 17-18).

\subsection{Effects on Robustness Profiles by Changing Weights in the Robustness Cost Function}

Trade-offs between satisfying different aspects of robustness can be balanced through changing the weights in the robustness cost function. In this section, the controller structure is unchanged, and the weights for the pitch-rate settling-time metric $I_{5 q_{-} q T_{s}}$, roll-rate settling-time metrics $I_{3 p_{-} p T_{s}}$ and $I_{5 p_{-} p T_{s}}$ are increased to 10 . The new design based on the cost function with modified weights is obtained as

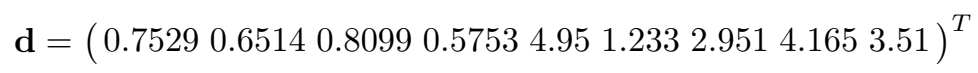




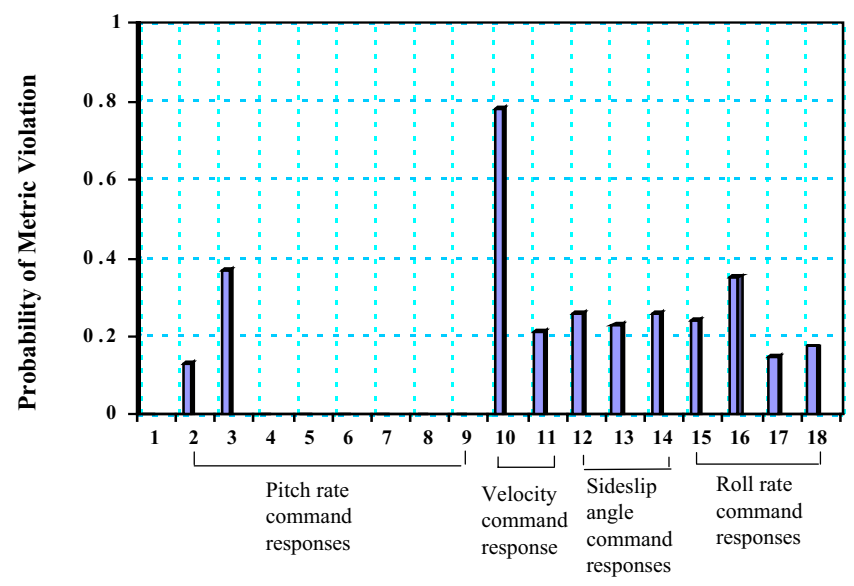

Fig. 8. Robustness profile of the present controller for the HIRM challenge.

Figure 9 shows the variations in the robustness profile of designs due to different weights in the robustness cost function. In Fig. 9, white bars (weight_1) represent the probabilities of violating design metrics for the design in Eq. 110, and dark bars (weight_2) denote the probabilities for the design in Eq. 111. Figure 9 shows that the probabilities of violating $I_{5 q_{-} q T_{s}}, I_{3 p_{-} p T_{s}}$, and $I_{5 p_{-} p T_{s}}$ (Metrics 3, 15 and 16) have decreased by almost two thirds. The probability of violating $I_{3 q_{-} q T_{s}}$ (Metric 2), and the probabilities of violating $I_{3 p_{-} q t}$ and $I_{5 p_{-} q t}$ (Metrics 17-18) have fallen to zero. However, the improvement in robustness for these metrics is achieved at the expense of increasing the probability of violating some other metrics. It is shown that the probabilities of violating requirements in sideslip-angle command responses (Metrics 12-14) are doubled, and the probability of violating the settling-time requirement in the velocity command response (Metric 10) has increased, too.

This comparison illustrates the limitation of redesign within a fixed controller structure. Changing cost function weights can improve specific responses, but it may do so at the expense of degrading the robustness of other responses. Comparing the original design vector (110) with the revised design vector (111), we see that the improved pitch and roll-rate responses led to 
higher airspeed and sideslip-angle damping, lower airspeed bandwidth, and stiffer yaw rate response. Further improvements would require revisions to the specified structures for slow and fast controllers.

$\square$ Weight_1 $\square$ Weight_2

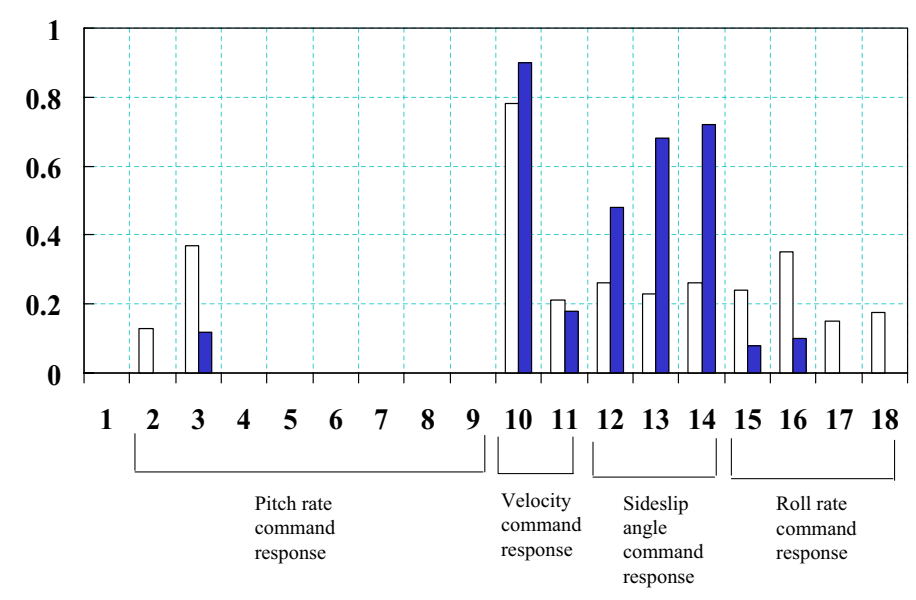

Fig. 9. Comparison of the robustness profiles of two designs with different weights in the robustness cost function.

\section{Conclusion}

Stochastic robustness analysis and synthesis break the computation complexity barrier suffered by deterministic worst-case approaches; Monte Carlo simulation and randomized search have polynomial complexity in computation. Instead of trying to guarantee that stability and performance specifications are satisfied for the worst-case scenario, the stochastic approach minimizes the likelihood of violating design requirements in the presence of expected variations in plant parameters. By focusing on the uncertainties most likely to occur in real engineering problems, the stochastic approach avoids undue conservativeness that could sacrifice nominal performance, cause extra controller complexity, and increase the possibility for control saturation. With Monte Carlo evaluation of probabilities of violating design metrics as an inherent feature of the control design process, a wide range of design specifications can be taken into account. Randomized algorithms such as genetic algorithms 
allow efficient tuning of design parameters for control problems formulated in a general and realistic fashion. The robustness profile of the final design and the choice of weights in the cost function provide sufficient information and flexibility for engineers to make tradeoffs between satisfying different aspects of robustness.

A stochastic robust nonlinear control design methodology is proposed by combining probabilistic robustness with feedback linearization (nonlinear dynamic inversion). The proposed approach is demonstrated through two design examples for robust flight control systems, where the high-fidelity models contain large dimensional uncertain parameters and complicated design specifications. The combination of stochastic robustness with nonlinear control design methodologies provides the ability to account for all significant nonlinearities and to produce better stability and performance robustness than linear robust control design with gain scheduling. The approach also reduces the complexity of control systems and the possibility of control saturation compared to the deterministic worst-case approaches to nonlinear robust control. It demonstrates engineering utility in addition to pure mathematical beauty, enhances the applicability of modern control theories, and reduces the gap between theory and practice.

\section{References}

1. J. Ackermann, D. Kaesbauer, and W. Sienel. Design by search. In 1st IFAC Symposium on Design Methodologies and Control Systems, 1991.

2. F. Amato, M. Mattei, S. Scala, and L. Verde. Design via LQ methods. In Robust Flight Control, A Design Challenge (GARTEUR). Lecture Notes in Control and Information Sciences 224, pages 444-463. Springer-Verlag, Berlin, 1997.

3. L. R. Anderson. Fine tuning of aircraft control laws using pro-Matlab software. AIAA-91-2600-CP, 1991.

4. T. W. Anderson and H. Burnstein. Approximating the upper binomial confidence limit. Journal of American Statistic Association, 62:857-861, 1967.

5. T. W. Anderson and H. Burnstein. Approximating the lower binomial confidence limit. Journal of American Statistic Association, 63:1413-1415, 1968.

6. D. M. Auslander, R. C. Spear, and G. E. Young. A simulation-based approach to the design of control systems with uncertain parameters. Journal of Dynamic Systems, Measurement, and Control, 104(1):20-26, 1982.

7. B. R. Barmish and C. M. Lagoa. On convexity of the probabilistic design problem for quadratic stabilizability. In Proceedings of the American Control Conference, pages 430-434, 1999.

8. G. W. Brown. Monte carlo methods. In E. F. Beckenbach, editor, Modern Mathematics for the Engineer. McGraw-Hill, New York, 1956.

9. G. Calafiore and B. Polyak. Fast algorithms for exact and approximate feasibility of robust LMIs. IEEE Transactions on Automatic Control, 46:1755-1759, 2001.

10. W. J. Conover. Practical Non-Parametric Statistics. J. Wiley \& Sons, New York, 1980. 
11. M. Cottrell, J.-C. Fort, and G. Malgouyres. Large deviations and rare events in the study of stochastic algorithms. IEEE Transactions on Automatic Control, 28(9):907-920, 1983.

12. E. J. Davison and A. Goldenberg. Robust control of a general servomechanism. Automatica, 11(5):461-471, 1975.

13. B. Escande. Nonlinear dynamic inversion and LQ techniques. In Robust Flight Control, A Design Challenge (GARTEUR). Lecture Notes in Control and Information Sciences 224, pages 523-540. Springer-Verlag, Berlin, 1997.

14. B. Etkin. Dynamics of Atmospheric Flight. John Wiley and Sons, New York, 1972.

15. Y. Fujisaki, F. Dabbene, and R. Tempo. Probabilistic robust design of LPV control systems. In Proceedings of the IEEE Conference on Decision and Control, pages 2019-2024, 2001.

16. E. G. Gilbert. Conditions for minimizing the norm sensitivity of characteristic roots. IEEE Transactions on Automatic Control, 29(7):658-661, 1984.

17. K. S. Gunnarsson. Design of stability augmentation system using $\mu$-synthesis. In Robust Flight Control, A Design Challenge (GARTEUR). Lecture Notes in Control and Information Sciences 224, pages 484-502. Springer-Verlag, Berlin, 1997.

18. N. Hovakimyan, F. Nardi, A. Calise, and N. Kim. Adaptive output feedback control of uncertain systems using single hidden layer neural networks. IEEE Transactions on Neural Networks, 13(6):1420-1431, 2002.

19. J. W. Howze and III R. K. Cavin. Regulator design with model insensitivity. IEEE Transactions on Automatic Control, 24(3):466-469, 1979.

20. K. Ito. On stochastic differential equations. Mem. Am. Math. Soc., 4, 1951.

21. S. Kanev and M. Verhaegen. Robust output-feedback integral mpc: A probabilistic approach. In Proceedings of the IEEE Conference on Decision and Control, pages 1914-1919, Maui, HI, 2003.

22. F. Kozin. A survey of stability of stochastic systems. Automatica, 5:95-112, 1969.

23. K. Krishnakumar and D. E. Goldberg. Control system optimization using genetic algorithms. Journal of Guidance, Control, and Dynamics, 15(3):735-740, 1992.

24. H. J. Kushner. Stochastic Stability and Control. Academic Press, New York, 1967.

25. P. I. Kuznetsov, R. L. Stratonovich, and V. I. Tikhonov. Non-Linear Transformations of Stochastic Processes. Pergamon Press, Oxford, 1965.

26. C. M. Lagoa, X. Li, and M. Sznaier. On the design of robust controllers for arbitrary uncertainty structures. In Proceedings of the American Control Conference, pages 3596-3601, 2003.

27. K. B. Lim and J. L. Junkins. Probability of stability: New measures of stability robustness for linear dynamical systems. J. of Astro. Sci., 35(4):383-397, 1987.

28. J.-F. Magni, S. Bennani, and J. Terlouw. Robust Flight Control, A Design Challenge (GARTEUR). Lecture Notes in Control and Information Sciences 224. Springer-Verlag, Berlin, 1997.

29. J. A. Markerink. Design of a robust scheduled controller using $\mu$-synthesis. In Robust Flight Control, A Design Challenge (GARTEUR). Lecture Notes in Control and Information Sciences 224, pages 503-522. Springer-Verlag, Berlin, 1997. 
30. C. I. Marrison. The Design of Control Laws for Uncertain Dynamic Systems Using Stochastic Robustness Metrics. PhD thesis, Princeton University, 1995.

31. C. I. Marrison and R. F. Stengel. Stochastic robustness synthesis applied to a benchmark control problem. International Journal of Robust and Nonlinear Control, 5(1):13-31, 1995.

32. C. I. Marrison and R. F. Stengel. Design of robust control systems for a hypersonic aircraft. Journal of Guidance, Control, and Dynamics, 21(1):58-63, 1998.

33. I. Miller and J. E. Freund. Probability and Statistics for Engineers. PrenticeHall, Englewood Cliffs, 1977.

34. B. S. Morgan, Jr. Sensitivity analysis and synthesis of multivariable systems. IEEE Transactions on Automatic Control, 11(4):506-512, 1966.

35. T. Motoda, R. F. Stengel, and Y. Miyazawa. Robust control system design using simulated annealing. Journal of Guidance, Control, and Dynamics, 25(2):267$274,2002$.

36. E. Muir. Robust flight control design challenge problem formulation and manual: The High Incidence Research Model (HIRM). In Robust Flight Control, A Design Challenge (GARTEUR). Lecture Notes in Control and Information Sciences 224, pages 419-443. Springer-Verlag, Berlin, 1997.

37. E. Muir. The robust inverse dynamics estimation approach. In Robust Flight Control, A Design Challenge (GARTEUR). Lecture Notes in Control and Information Sciences 224, pages 541-563. Springer-Verlag, Berlin, 1997.

38. G. Papageorgiou, K. Glover, and R. A. Hyde. The $H_{\infty}$ loop shaping approach. In Robust Flight Control, A Design Challenge (GARTEUR). Lecture Notes in Control and Information Sciences 224, pages 464-483. Springer-Verlag, Berlin, 1997.

39. A. Papoulis. Probability \& Statistics. Prentice-Hall, Englewood Cliffs, 1990.

40. C. Pierre. Root sensitivity to parameter uncertainties: A statistical approach. International Journal of Control, 49(2):521-532, 1989.

41. B. T. Polyak and R. Tempo. Probabilistic robust design with linear quadratic regulators. Systems $\&$ Control Letters, 43:343-353, 2001.

42. L. R. Ray. Stochastic Robustness of Linear Multivariable Control System: Towards Comprehensive Robust Analysis. PhD thesis, Princeton University, 1991.

43. L. R. Ray and R. F. Stengel. Application of stochastic robustness to aircraft control systems. Journal of Guidance, Control, and Dynamics, 14(6):1251-1259, 1991.

44. L. R. Ray and R. F. Stengel. Stochastic measures of performance robustness in aircraft control systems. Journal of Guidance, Control, and Dynamics, pages 1381-1387, 1992.

45. L. R. Ray and R. F. Stengel. Computer-aided analysis of linear control system robustness. Mechatronics, 3(1):119-124, 1993.

46. L. R. Ray and R. F. Stengel. A Monte Carlo approach to the analysis of control system robustness. Automatica, 29(1):229-236, 1993.

47. W. M. Schubert and R. F. Stengel. Parallel stochastic robustness synthesis for control system design. IEEE Transactions on Control Systems Technology, 6(6):701-706, 1998.

48. A. A. Schy and D. P. Geisy. Multiobjective insensitive design of airplane control systems with uncertain parameters. In Proceedings of the AIAA Guidance, Navigation and Control Conference, 1981. 
49. J. D. Shaughnessy, S. Z. Pinckney, J. D. McMinn, C. I. Cruz, and M.-L. Kelley. Hypersonic vehicle simulation model: Winged-cone configuration. Technical report, NASA TM 102610, 1990.

50. D. D. Siljak. Parameter space methods for robust control design: A guided tour. Technical Report EECS-031588, University of California, Santa Clara, 1988.

51. R. C. Spear. The application of Kolmogorov-Rényi statistics to problems of parameter uncertainty in systems design. International Journal of Control, 11(5):771-778, 1970.

52. R. F. Stengel. Some effects of parameter variations on the lateral-directional stability of aircraft. Journal of Guidance and Control, 3(2):124-131, 1980.

53. R. F. Stengel. Optimal Control and Estimation. Dover, New York, 1994.

54. R. F. Stengel and L. R. Ray. Stochastic robustness of linear time-invariant control systems. IEEE Transactions on Automatic Control, 36(1):82-87, 1991.

55. K. C. Tsai and D. M. Auslander. A statistical methodology of designing controllers for minimum sensitivity of parameter variations. Journal of Dynamic Systems, Measurement, and Control, 110(6):126-133, 1984.

56. Q. Wang. Stochastic Robust Control of Nonlinear Dynamic Systems. PhD thesis, Princeton University, 2001.

57. Q. Wang and R. F. Stengel. Searching for robust minimal-order compensators. ASME Journal of Dynamic Systems, Measurement, and Control, 123(2):233236, 2001.

58. B. Wie and D. S. Bernstein. A benchmark problem for robust control design. In Proceedings of the American Control Conference, pages 961-962, 1990.

59. E. Yaz. Deterministic and stochastic robustness measures for discrete systems. IEEE Transactions on Automatic Control, 33(10):952-955, 1988.

60. G. E. Young and D. M. Auslander. A design methodology for nonlinear systems containing parameter uncertainty. Journal of Dynamic Systems, Measurement, and Control, 110(6):126-133, 1984. 